$\square$

\section{$\square$}

$\Delta$

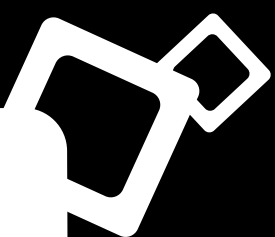

DOCUMENTOS

INSTITUCIONALES
* Propuesta de conversión del Centro de Investigación Científica (CINC) en Centro de Investigación Científica y Estudios Es. tratégicos de Seguridad (CINCEES). 
$\square$

$\square$

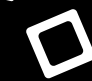

* Proposal to convert the Center for Scientific Research (CINC) into a Center for Scientific Research and Strategic Security Studies (CINCEES) 


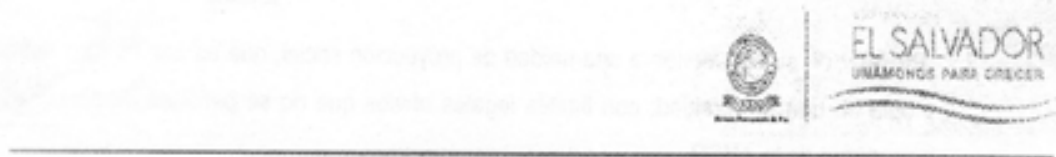

MEMORANDO MCINC 51-09-2015

Para: Sr. Director General ANSP

C.C: Sr. Subdirector Ejecutivo

De: Coordinador Principal CINC-ANSP

Asunto: Propuesta estratégica de conversión del CINC en tanque de pensamiento (CINCEES)

Fecha: 21 de Septiembre de 2015.

Han transcurrido casi dos años desde que este servidor concluyó y presentó a la instancia que preside el primer informe estratégico intitulado: "Apuesta estratégica para el desarrollo futuro del Centro de Investigación Cientifica CINC. ANSP". Documento de 16 páginas entregado con fecha 30 de octubre de 2013.

En aquel momento se planteó como propósito general, orientar estratégicamente los esfuerzos de cualificación, fortalecimiento y potenciación del Centro de Investigación Cientifica; identificando areas criticas y desafios por enfrentar.

Este nuevo esfuerzo y que constituye el segundo informe estratégico, recoge una propuesta de largo alcance intitulada: "Propuesta de conversión del Centro de Investigación Cientifica (CINC) en Centro de Investigación Cientifica y Estudios Estratégicos de Seguridad (CINCEES)"; los objetivos de este documento son entre otros:

1. Salvaguardar una experiencia exitosa en materia de organización para la gestión cientifica y su producto elite, la revista especializada "Policia y Seguridad Pública" que se caracteriza por el formato de un journal cientifico de seguridad tipo anglosajón.

2. Prevenir de las propuestas de reasimilación del CINC-ANSP en otras unidades o divisiones cargadas de burocratismo y ausencia de mecanismos meritocráticos, que constituyen problemas de larga data y que continuan presentes aủn durante la marcha del proceso de acreditación como Instituto de Educación Superior (IES).

Centro de Investigación Cientifica CINC-ANSP

Avenids Melvin Jones frente a Parque San Martin, Santa Tecla, La Libertad

juan.moraleseansp.gob.sv

Tel. 2565.6667 

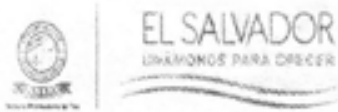

3. Prevenir de su conversión a una unidad de proyección social, que es una función estratégica propia de una universidad; con limites legales obvios que no se perciben, ni ponderan en la comunidad de la ANSP.

4. Prevenir de su conversión en una unidad, que se carga con lineas de investigación marginales del ámbito curricular y educativo de nivel básico. Un rubro o tendencia de potencial anulación de la fuerza internacional y extra institucional que se ha desarrollado en el seno del CINC.

5. Prevenir de la caida de los estándares internacionales cumplidos y reconocidos tanto en distintas bases de datos académicas como en comunidades de expertos de entidades públicas y privadas, de los ámbitos nacionales e internacionales.

6. Prevenir de toda iniciativa, tendencia o dinámica conducente a su reabsorción organizativa, cambio de sus objetivos y en general de toda aquella reestructuración que desnaturalice la misión y visión tanto del Centro de Investigación Cientifica, asi como las de la revista "Policia y Seguridad Pública".

De todo lo anterior se desprende, que en el ámbito extra institucional y en lo que concierne a los interlocutores involucrados, en este caso el Ministerio de Educación (MINED) y el Consejo Nacional de Ciencia y Tecnologia (CONACYT); no cuentan con los programas, agendas y lineas estratégicas para la investigación en seguridad, ello plantea una falla de origen que imposibilita la construcción temprana de un direccionamiento nacional en dicha materia, debilitándose por lo tanto en legitimidad y autoridad. De esta lectura, se hace hincapié en la adopción de aspectos claves. para la viabilidad de esta propuesta estratégica y sus respectivos términos y orientación, por ello el proyecto CINCEES busca entre otros objetivos:

1. Atender asertivamente las dinámicas de la seguridad en el pais y la región.

2. Innovar en las respuestas de seguridad en cuanto a diseño y direccionamiento.

3. Alimentar estructuras nacionales ampliadas que conciernen a las comunidades del ámbito de la seguridad nacional (seguridad pública, defensa y relaciones exteriores). Con énfasis en la primera.

4. Dotar de nuevo estatuto juridico a la estructura sucesora del CINC - ANSP, como unidad organizativa staff y diferenciada en autonomia de las jerarquias del área de estudios.

Centro de Investigacion Cientifica CINC.ANSP Avenida Melvia Jones frente a Parque San Martin, Santa Tecla, La Libertad iuan.moraleseansp. gob.sv Tel. 2565-6667 


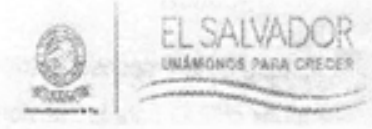

5. Generar nuevos rangos de plazas y umbrales económicos-salariales al equipo de planta.

6. Proveer de inversiones estratégicas crecientes en los fondos de investigación de una estructura con dimensiones de CINCEES, que incluya el establecimiento de una politica de administracion financiera.

7. Propiciar un crecimiento ordenado de planta de investigadores y otros colaboradores, concibiéndolo como una imversión de pais, experiencia apoyada en buenos resultados del pasado reciente y orientada por los imperativos de la sequridad.

8. Racionalizar propuestas y modalidades intermedias del formato de tanque de pensamiento, siempre y cuando éstas se sustenten en la gestión cientifica de alto nivel, la producción investigativa de nivel politico-estrategico y se desvincule de niveles básicos de la estructura que pondrian en riesgo sus capacidades.

9. Promover esta propuesta como apuesta fuerte en el marco de los cambios y transición de la ANSP a un Instituto de Educacion Superior (IES).

Junto al documento central de esta propuesta, encontrará también dos anexos. Uno concierne a los resultados de la evaluación general de Latindex (Sistema Regional de Información en Linea para Revistas Cientificas de América Latina, el Caribe, Espańa y Portugal); hizo de la revista "Policia y Seguridad Pública" y que la introdujo hacia indizaciones más especializadas. Por su parte el otro anexo lo constituye una sección de un estudio estratégico reciente de este servidor, en el que se analizo la experiencia del Centro de Investigación Cientifica y que se incluyb en el númera seis (6) de la revista que desamolló la temática "Prospectiva de Seguridad".

Asi tambièn a lo largo y ancho de la propuesta, encontrará citas textuales, pié de páginas y otras referencias de distintos estudios, cartas del editor y discursos oficiales; todos de este servidor, no por ningún objetivo inútil de auto promoción, si no como recursos de apoyo que autentican la visión del proyecto CINC, las apuestas que se han materializado y la fuerza con la que se han desarrollado en sus distintas etapas.

Centro de investigación Cientifica CINC-ANSP 

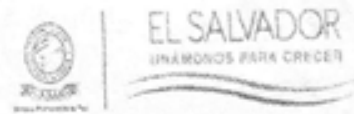

La presente propuesta estratégica recoge los niveles hacia donde deben generarse impactos, las áreas criticas para la transformación de la seguridad, la superación de los factores restrictivos que median entre las iniciativas emergentes en esta materia, pero sobre todo, quizá ésta sea la única oportunidad para fortalecer $y$ garantizar un esfuerzo que ha sido pionero y vanguardista, como proyecto avanzado anclado en una visión de pais, como una expresión notable de lo que debe ser la politica de Estado, aunque lo sea a pequena escala y las circunstancias asi to hayan permitido en cuanto a sus alcances, su vocación de estabilidad y permanencia más allá de los vaivenes politicos y los anquilosamientos institucionales.

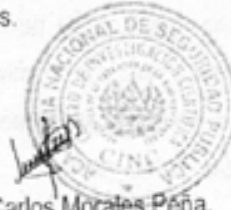

Juan Carlos Morates Pena.

Coordinador principal

CINC-ANSP 


\title{
Propuesta de conversión del Centro de Investigación Científica (CINC) en Centro de Investigación Científica y Estudios Estratégicos de Seguridad (CINCEES)
}

\begin{abstract}
Propósito general
La Academia Nacional de Seguridad Pública es la única entidad estatal que sirve una oferta educativa con la que se forma a los profesionales de la seguridad.

Constituye el nicho institucional idóneo en el cual El Salvador puede hacer una inversión estratégica importante para potenciar al centro de investigación que existe desde 2011 y establecer asi las bases sobre esta estructura para desarrollar un tanque de pensamiento para la seguridad; en el que se habilite y extienda la plataforma de estudios estratégicos que ya opera con algunas líneas de trabajo. Ello contribuiria decisivamente al quehacer del funcionariado de este rubro sobreexigido y ampliamente demandado socialmente.
\end{abstract}

No existe institución alguna, ni en el ámbito privado, ni en el público, donde funcione un ente con la suficiente oportunidad para generar autonomía propiciando capacidades de gestión de la investigación en materia de seguridad.

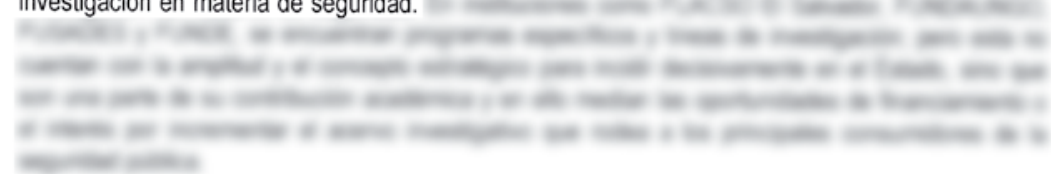

Estos aspectos, lo diferencian del modelo de tanque de pensamiento, que es un reservorio institucional muy distinto; porque hay experiencias escasas en el país con la lógica de este diseño.

Al interior de un tanque de pensamiento, se confeccionan estudios y propuestas avanzadas; que cuentan con amplios umbrales de aplicación en un sector 0 área especifica de trabajo en el Estado. No se trata de productos académicos, en su carácter de insumos para la discusión pública aunque lo incluye en su justa dimensión; sino de instrumentos de impacto decisivo en la conducción de las materias especializadas de la vida estatal.

En el caso del proyecto (CINCEES); que es en el que se basa esta propuesta, convergen distintos aspectos: En primer lugar factores restrictivos de entorno, en segundo lugar los niveles de incidencia; en tercer lugar las áreas críticas de impacto; en cuarto lugar la evaluación de las políticas editoriales y de gestión científica, en quinto lugar el patrimonio académico CINC; en sexto lugar una vital retroalimentación del proyecto desde propuestas anteriores y en séptimo y último lugar la contrapropuesta del CINC ante el proyecto del Instituto de Educación Superior (IES).

Pero antes de presentar más ampliamente estos aspectos, es menester identificar los factores restrictivos de realidad y entorno que constriñen la orientación de la seguridad pública y de cómo ello influye en la construcción de agendas institucionales de investigación. 


\section{Factores restrictivos}

A. Los defectos de fábrica del Instituto de Educación Superior (IES)

Por ser este esfuerzo institucional, una iniciativa vinculada al formato de universidad; y que constituye el nicho natural de orientación por parte de la máxima autoridad educativa del pais, (el MINED en este caso); el proyecto por si mismo descartó prematuramente la posibilidad de un diseño ad hoc, coherente con la esencia sistémica de la institución y la función tan especializada que recoge. Es decir, las instituciones educativas de nivel superior en materia de seguridad y defensa. por su naturaleza pueden adoptar dinámicas organizativas universitarias, pero basarlas exclusivamente en esto las atrofia en su diseño organizacional porque no construye relaciones estratégicas para el desarrollo constante del campo o sector en el que se inscriben. Generalmente en una universidad, una tesis de grado e incluso de un programa de maestria, no se funda en el imperativo de publicación, en el de difusión y establecimiento de áreas y sectores claves por alcanzar, por la ausencia de programas de investigación institucionales, en cuyos objetivos se plantearia la capitalización de estos productos. La extensión del quehacer y la vocación de aporte de una universidad hacia afuera se basa en el espacio de proyección social, que es diverso e inestable en el tiempo; por responder a lineas de trabajo coyunturales y en las que no se encuentran comprometidas a toda regla los presupuestos para el financiamiento investigativo y las capacidades instaladas del recurso humano, que suele fluir en una lógica de relevo según los alumnados avancen hacia los procesos criticos de graduación y cumplimiento de requisitos administrativos finales.

El defecto de fábrica del IES, tambièn tiene una falla exterior; y èsta se refiere a que ni el MINED (Ministerio de Educación), ni el CONACYT (Consejo Nacional de Ciencia y Tecnologia); cuentan con lineas estratégicas de investigación para los sectores de seguridad, defensa y relaciones exteriores: porque sus comunidades de expertos desconocen las dimensiones de la oferta educativa y de especialización de estos entes públicos; de tal manera que ello ha traido como resultado que las lineas estatales en materia de desarrollo cientifico y tecnologico prescritas y operativas sean insuficientes para orientar la creación de sistemas de investigación institucionales, programas de investigación, politicas de financiamiento para la investigación entre otras y para el caso de éstas entidades. Todo lo anterior trae consigo la adhesión a campos envolventes y más globales como las ciencias sociales, que conducen hacia la concepción deficitaria de prioridades y diagnósticos que complican o restringen el desarrollo de disciplinas cientificas no convencionales propias de las instituciones de seguridad que cuentan con sus respectivos objetos de estudio, programas de investigación, comunidad de investigadores, recursos metodológicos y modelos de análisis propios e incluso paradigmas explicativos ${ }^{1}$ en evolución constante. Por ello pensar estratégicamente en un diseño adhoc, es vital y significa reconocer la identidad de la comunidad donde emerge, ponderar en su justa dimensión su función estratégica dentro del Estado; pues recuérdese que la policia forma parte del aparataje estatal; mientras que la universidad tambienn puede formar parte de éste pero no necesariamente homologa su quehacer a la actividad estatal, en aspectos de gestión académica y

\footnotetext{
LCuando un parad gma de cualquiar campo discolinario es peceoninante y no ha sido desplazado como modelo de referencia en las comunidades de especialistak, se dice que no ha ocurrido una revolución clentifica o paradigmatica. Sin embargo no basta que este sea desechado, sino que otro lo sustituya, se entronice y sirva como nuevo modelo de referencla dominante. Esta es la principal razón, por la cual exisse debate entre los adherentes de los paradgmas tradicionales con fuerte presencia en el entorno tales como: Las teorias da la securitización, la teoria de la securidad nacional, los estudios clasicos de securidad, realismo y neorrealismo como principales. Y por su parte otres emergentes de determinado desarrollo teorico pero deficientes en recurvos metodologices de operativizacion de categorias e indicadores para el planeo de la ata politica y la estrategia de iatervención. Aca se encuentran las escuelas inglesa y de Copenapue, el constructirismo, los estud os criticos de la seguridad, el post estructuralismo entre otros.
} 
cientifica. Este último ente puede estar distribuido y afincando en la sociedad y responder a los estimulos del mundo privado

En el caso especifico de de la ANSP, ésta no es una universidad; asi como no lo son el Instituto de Educación Superior para la Formación Diplomática (IEESFORD) del Ministerio de Relaciones Exteriores; la Escuela Superior de Economia y Negocios (ESEN); ni el Colegio de Altos Estudios Estratégicos (CAEE) en el Ministerio de la Defensa Nacional. Son instituciones de educación superior, pero cuentan con un diseño ad hoc, con un área disciplinaria de alta especialización. La dificultad estriba en que de acuerdo a expertos en educación superior y coincido con ellos, es que cumplir con los parámetros inherentes a una universidad, resultará imposible para estas entidades. Los pares evaluadores del Ministerio de Educación (MINED), reduciràn su trabajo a insistir una y otra vez a lo estipulado en la Ley General de Educación Superior, que se encuentra sobrepasada por la realidad de los institutos especializados. Sólo será procedente una reforma en la misma con las enmiendas y desarrollo de contenido respectivo para institutos superiores que no tienen el formato de una universidad. Asi tambien será necesario que todas las entidades con la naturaleza apuntada, tanto públicas como privadas, lideren iniciativas de reforma legal substanciales. Los institutos especializados u otros con el diseño de tanque de pensamiento no tienen "proyección social"; porque su trabajo se dirige hacia objetivos nacionales que el pais recoge en su legalidad e institucionalidad, de alli deriva la materialización de un servicio superior en el ámbito del alto gobierno y esto es consubstancial con el servicio a la nación.

\section{B. La complejización del entorno de seguridad}

La realidad del entorno en materia de seguridad, ha sobrepasado estimaciones previstas; golpeado a las politicas públicas del sector, sobreexigido al Estado y puesto a prueba el desempeno y rendimiento del aparato policial. Esta situación condujo a que la concepción investigativa en el CINC planteara desde sus origenes un nivel politico-estratégico para la producción de estudios especializados.

El Salvador registra niveles de violencia sin precedentes, teniendo ello como caracteristica la sofisticación organizada y operativa del mundo criminal. Este fenómeno tiene connotación regional y es influido por la actividad ilicita a nivel global. Los campos de acción para el Estado, son más complicados y diversos; la capacidad de ejercicio soberano o de reconstruoción de la autoridad se restringe por las presiones de la globalización y los niveles de infiltración, faccionalización, corrupción y cooptación al interior del aparato público². Lo anterior se suma a la escasez de politicas públicas y diseño estratégico, que trazan en conjunto importantes conexiones con la economía, la sociedad, la recaudación de impuestos, la inversión pública entre otros rubros vitales.

El entorno de seguridad, trae consigo las necesidades de readecuacion en el Estado y la sociedad; no resulta factible ensayar respuestas cuyos resultados podrian ser desastrosos, escasos ó inciertos; emerge acá un imperativo de verdadera ingenieria creativa e innovadora capaz de sobrepasar todo inmovilismo y por lo tanto ello incluye el empoderamiento de los distintos actores en el Estado y la sociedad en la construcción de iniciativas para la seguridad.

\footnotetext{
Vease Morales Pels, Juan Carlos. Nuevos empoderamientos criminales y cesafes gobales para los Estados del Trineulo Norte y
} Centroamericano y Misico". Revista "Policia y Seguridad Pública", Ano 2, Vol. 1, enero-junio 2012, pigs. 221-237 


\section{Centro de Investigación Cientifica CINC 2015}

\section{La Politica de Seguridad como Politica de Estado}

Durante toda la post guerra, el pais no ha tenido una politica de seguridad pública que haya sido concebida a nivel de Estado. La tendencia prevaleciente ha sido la de nivel gubernamental, liderada principalmente por la administración de turno en el Ejecutivo; ente que la hecho fluir y empoderar hacia las instituciones que se encuentran bajo su jurisdicción como la Policia Nacional Civil (PNC), la Dirección General de Centros Penales, la Academia Nacional de Seguridad Pública (ANSP) y otras afines débilmente diseñadas sucesoras del entonces Consejo Nacional de Seguridad Pública (CNSP). El asunto aca es que el Estado no lo conforma sólo el órgano Ejecutivo, sino también las otras dos ramas (Legislativa y Judicial) y éstas participan sistémicamente en la hechura de la seguridad pública de un pais. Esta imposibilidad ha sido producto de conceptos de escaso alcance basados en la coordinación interinstitucional y no en auténticas relaciones estratégicas, que traen consigo un desempeño bajo la lógica de la unicidad, la complementariedad, la reingenieria institucional y la reinvención funcional.

Los otros aspectos comunes que to ha impedido es el conflicto de paradigmas en materia de seguridad, la fluctuación de posturas, los sentidos de prioridad, la idea de intervención territorial, la percepción de amenaza, la concepción de los estados de salida y la gestión de los largos plazos; son factores que influyen en la conducción de la seguridad, en el liderazgo estratégico institucional y en las opciones que los gobernantes de tumo tienen en las coyunturas de ciclos de crisis de la seguridad pública.

De todo lo anterior se desprende que una ANSP que evoluciona hacia una forma de universidad policial, debe contar con un tipo de investigación dinigida a la transformación del sector seguridad y de ésta en todas sus connotaciones; debe movilizar el modelo de los estudios avanzados, que les es inherente a las estructuras que se conciben como tanques de pensamiento. Esto es en si una aportación decisiva para el pensamiento estratégico en el pais, especificamente el vasto campo de la seguridad.

\section{La revisión estratégica de seguridad}

Se entiende por revisión estratégica al proceso mediante el cual un sector especializado y especifico del Estado es evaluado y analizado decisivamente para los efectos de su reinvención o transformación, por la influencia de los desafios futuros que le conciernen y por la presión del entorno complejo en el que se inscribe.

Este proceso es liderado por una administración presidencial y el gabinete responsable que ejerce funciones en el área critica en cuestión, para el caso que nos ocupa el sector seguridad. Haciendo extensiva la consulta calificada a varios niveles: Siendo los primeros las comunidades de seguridad, defensa e inteligencia. En este ejercicio intervienen y para ilustrarlo mejor expresidentes, ex directores de los servicios de inteligencia, ex directores de las academias policiales, ex directores de policias, ex directores de centros penales; para ello se establecen metodologias ad hoc según los requerimientos del proceso y se cubre en una primera etapa las problemáticas de los niveles de decisión estratégica y politica estratégica.

La substancia de esta etapa es la extracción de lecciones, aprendizajes, particularidades de los entornos de seguridad e inventarios de éxitos y fracasos de cada periodo de gestión. Esta dinámica se sigue desarrollando a otros niveles y personeros que van incorporando los escenarios tácticos. operativos y otros. 


\section{Centro de Investigación Cientifica CINC 2015}

La revisión estratégica propicia la radiografia de las relaciones entre los órganos estatales, atendiendo materia de gestión de la seguridad en otra etapa de evaluación. Puede incluir otros esfuerzos similares que recogen la perspectiva y el análisis de numerosos actores en otras instituciones, en la sociedad y el territorio; todo ello mediante el planeo de eventos dirigidos en los que se procesan las consideraciones y evaluaciones de los mismos; por parte de grupos de trabajo gubernamental. Es decir limita el dialogo sectorial como escenario preeminente, en cuanto este se convierte en el primario y exclusivo. Porque los insumos de estos niveles tienen implicaciones operativas y a lo sumo tácticas, pero no contribuyen decisivamente a la concepción estratégica de la seguridad; la que se ubica en el ámbito de la alta politica. Por cuestiones de seguridad y alta sensibilidad de los temas a tratar, en estos espacios no deben coincidir junto a los agentes del Estado con otros actores distantes a la comunidad de seguridad. El nivel de consulta con base estratégica es la que propicia las auténticas evaluaciones especializadas porque convoca a la comunidad de analistas estratégicos, planificadores, asesores y formuladores de estrategia.

\section{E. El agujero del Asesoramiento Estratégico}

Los paises de estas latitudes no cuentan con un verdadero asesoramiento estratégico, entendiéndose éste como el conjunto de competencias especializadas que basadas en la experiencia y el conocimiento de herramientas de gestión y caracteristicas de los problemas; da vida a una pujante comunidad en donde existen relevos generacionales ordenados y se produce una transferencia de capacidades en el marco de la funcionalidad de una escuela de alto nivel y programas institucionales. El asesor de seguridad de estos tiempos presenta tres aspectos que atrofian los imperativos de esta función estratégica vital. En primer lugar no tiene experiencia y ello les impide contar con capacidad de rectificación, los vuelve ineficaces para comprender la connotación de la historia y su influencia para afectar ciclos recurrentes de crisis en materia de seguridad $e$ incluso de otra indole. Al no ser el elemento experiencia clave para la formación o reclutamiento de asesores, es porque también no han sido parte de relevos generacionales, se ha padecido de la ausencia de mentorias politicas y estratégicas de orden fundamental.

En segundo lugar los gobiernos cuentan con perfles tecnocráticos del asesor, esto crea dependencia en demasia de la formación académica, que generalmente no se aproxima a los ámbitos y requisitos definidos. Se empobrece por la excesiva academización y esto le resta sentido de practicidad para generar o recomendar soluciones en áreas criticas. El producto de asesoramiento de esta tipologia se reduce a la apreciación macro muy bien estructurada, pero es incapaz de innovar en soluciones de alta incidencia en los sistemas de trabajo de los tomadores de decisiones y por lo tanto sin alcance para propiciar quiebres de tendencias en el entorno.

Y en tercer lugar se carece de "Escuela" el reservorio donde deben formarse los analistas, los estrategas de seguridad, los asesores; ${ }^{3}$ contando con diseños educativos prácticos, con la filosofia aprender-haciendo; bajo el concepto de alto y bajo gobierno; con programas de investigación y planes de estudios especializados. Para que el sujeto que se desempeñará en el sector, œnozca áreas criticas y los desafios de la cultura estratégica en todo espacio estatal.

\footnotetext{
'Véase Morales Peña, Juan Carlos. "El pollola estrotégico frente a la crisis futura y las paradojas del Estado en la reconstitución de su autoridad". Aौ̄o 3, Vol. 1, mayo - octubre 2013, pág. 188
} 


\section{F. La oferta educativa}

Las academias policiales constituyen los entes públicos responsables de la formación policial; sin embargo, este subsector que sirve y confecciona la oferta educativa y de especialización en general está aquejado por una serie de problemas estructurales. La oferta educativa no sólo se orienta a la producción de policias, en lógica fordista, aunque sea natural que asi sea cuando en el entorno de seguridad hay presiones y amenazas criminales de alta complejidad.

Aún sin lo anterior, la oferta educativa sigue siendo deficiente, porque sigue contando con plantas docentes cuya experiencia existe como resultado de largas permanencias laborales en las instituciones y no porque sus expectativas hayan coincidido con auténticos programas de especialización y formación continua. Con lo anterior, prevalece el inmovilismo y los modus vivendi negativos que atrofian a todo nivel la transferencia de conocimientos.

La oferta también es insuficiente, cada policia formado no cuenta con el minimo aceptable de horas lectivas y carga académica por superar.

En el caso de paises como El Salvador y otros centroamericanos, este esfuerzo de modemización educativa de nivel superior, se ve restringido por el perfil colectivo de las comunidades docentes. A la falta de competencias se suma, la demanda de elevada formación académica y de alta especialización. Este es el desafio estratégico más sobrecogedor para las academias de policias. Ejercen presión además, las amenazas complejas del entorno; y por ende cualquier oferta educativa debe fundarse en pivotes estables, en los que se haya trabajado directrices inteligentes para la gestión de plazos y conceptos estratégicos que propicien capacidades de reinvención de toda la organización pública.

\section{Niveles de incidencia y tipologia de impactos.}

Los programas de investigación activos en un tanque de pensamiento en seguridad, deben generar productos avanzados, capaces de propiciar impactos críticos e influencias decisivas en cuatro niveles importantes de la actividad estatal. Estas conciernen a los ámbitos institucionales, estratégicos, táctico - operativo y estructural. A continuación me refiero al alcance y dimensiones de los mismos.

\section{NIVELES}

Nivel institucional: Se entenderá como el espacio interior de un ente público para transformarse en el que se incluyen entre en otros aspectos fundamentales: Cultura organizacional, la planta laboral, los sistemas de trabajo, el clima organizacional, las funciones estratégicas, las competencias legales, su servicio público, su oferta sistémica especializada, su jurisdicción, sus relaciones intrasistemáticas y extrainstitucionales, su concepción cooperativa, su liderazgo estratégico, su producción investigativa, su estructura salarial, su potencial de innovación, sus iniciativas de transformación, su historia, el talento humano, la moral y estructura de incentivo entre los miembros, la carga de responsabilidades, su concepto de seguridad institucional, de documentos y de información, riesgo y salud ocupacional, capacitación y especialización de sus miembros. Asi también tiene implicaciones importantes en el ámbito decisional de titulares de cartera, consejos directivos, académicos o de administración. 


\section{Centro de Investigación Cientifica CINC 2015}

Nivel estratégico: Se entenderá como el espacio de implicación extrainstitucional y todo aquel de indole interior que se vincula a un desenvolvimiento en el entomo y que por lo tanto dinamiza ciclos de resultados y retroalimentación. Estos últimos se expresan con diversas connotaciones en rubros tales como: cambio de tendencia, gestion de coyunturas criticas, construccion de potencialidades, transferencias de capacidades, aseguramiento de condiciones; todas las cuales se sujetan a indicadores de progreso y otras unidades de medida o ponderación avanzada. En materia especifica concierne a rendimiento policial, a eficacia de politicas, a modemizacion del sector, a innovación de especialidades, a profesionalización policial, a fortalecimiento estatal, infuencia regional, perspectiva global, alianzas internacionales, asocios estratégicos públicos-públicos, asocios públicos-privados, relacionamiento estrategico, etc. Involucra decisores que incluyen ttulares, ministros, directores, presidentes, asesores, estrategas, planificadores y otros.

Nivel estructural: Se refiere a que los impactos son transversales en más de un sector especializado del Estado. Es decir, que las politicas públicas del sector seguridad y sus diseños se entrelazan con los ámbitos presupuestarios, fiscales, económicos y sociales. Que la influencia decisiva de los mismos estrecha la cooperación al interior del Estado, posibilita el relacionamiento estratégico, descomplica la gestión de todo un sector y resuelve los problemas planteados del entorno en una logica de progresividad e irreversibilidad. Sus expresiones más comunes es cuando se producen condiciones para formar asesores y analistas, para la revisión estrategica del sector, para el planeamiento de politica bajo el concepto de Estado y para la transdisciplinariedad evaluativa de un sector o área critica. Es decir trae consigo dinámicas y sinergias importantes con los actores estatales, poderes públicos, actores económicos, politicos y actores sociales.

Nivel táctico operativo: Concierne a impactos que en los planos de la gestion cotidiana o de proyecciones mensuales 0 anuales, determinan nuevos cursos de acciön, moldean las decisiones, propicia ajustes sobre la marcha, plantea umbrales asegurables en cortos y medianos plazos entre otros. Siendo estos últimos decisivos para la recuperación de confianza, restauración de autoridad, establecimiento del control territorial, retomo y aseguramiento de la paz social. Rehabilitación de la presencia estatal o construcción del Estado desde abajo, además de la capacidad de dispensación de un servicio esencial a la comunidad y la sociedad. Al respecto y en relación a los contenidos expuestos por este servidor en un estudio sobre el policía como actor estratégico apuntaba sobre la construcción de Estado desde abajo lo siguiente:

'En primer lugar el policia estrategico debe ser formado para que sea capaz de construir Estado desde abaja, su desenvolvimiento crientado a la instifucionalidad debe contnbuir de fal forma que pueda ser apreciado como un actor expresivo de la estafaidad para los imperativos y esfuerzos colectivos de la cohesión social. Significa que sea identificado como un interiocutor valido o legitimo para arbitrar en medio de las dinamicas de la confictividad social. Se trata más bien de un habilitante de institucionslided, de un tejedor de reglas y pautas de autoridad; dispensación de un servicio que cree coaliciones de apoyo local de todos aquellos que rechazan la prosencia de organizaciones criminales. Implica la garantia de extender en el tiompo y en of nodo ternitorial un factor presencial disuasivo que inhiba a otros. actores licitos en sus intentos por ocupar o lenar los vaclos de autoridad.

Significa que lus condiciones que se propician sean irreversibles, genere correlaciones sociales favorabies y abran el camino a dros miembros de la burocracia especialzade del Estado y que fienen base feritorial. Este esfuerzo incluye el desmantelamiento de las redes de apoyo comunitario de las estructuras delictivas, implicando ademós el atacue a sus redes de informantes y colacoradores. Conlieva al fomento del liderazgo en las operarios de la segurided y el empoderamiento de la población para tomar responsabiligd sobre sus propios asuntos. Esto va más alla del policia comuntario que enfatiza la construcción de puentes con el cludadano, y que también es necesaria, pero no se plantea cómo recuperario defintivamente para la legalidad o como extraerlo del socuestro social o cosptacín por grupos que 
han prevalecido con regles de la paz criminal o estimulado la no coservancia a las que dispensa el Estado" (Morales Perla, 2013 pág. 185]

Por su parte existe también la modalidad de construcción del Estado desde arriba, que en contextos de guerra se derivan de la implantación de sistemas de ocupación o de esfuerzos de reconstrucción en donde participan distintas naciones en coalición. Asi también contextos de posguerra, en donde la dinámica se ha situado más bien en la conducción de las reformas económicas desde el sector público. Al respecto se apuntaba:

-...En definitiva debe fenerse en cuenta que construir Estado desde ambe leva consigo una significativa inversión en ed sistema del ocupante para gestionar lo mejor posible la past guerra; condición que muchas veces es el inicio de otra más prolongeda y compleja por los anticuerpos que portan las tropas invascros. $Y$ que aún lograndose imponer una paz precaria para que las instituciones, el tejido social y productivo empresarial se reconstituyan y funcionen requerirán ailos de aportaciones financieras del exterior para conducirlas a capacidades de producción, exportación, recaudación de impuestos, inversión pública y social habilitación de econamia puiblica en otros aspectos y materias fundamentales.... [Marales Pella, 2013 pog. 198)]

\begin{abstract}
-... Debe acotarse que en el caso de los Estados fallidos y de los esfuerzos de reconstimacion que se ïevan a cabo en las mismos, la estrafegia primaria es la construcción de Estodo dosdo amibo y ello implica una serie de elementos y condicianes tales coma: Primero, propiciacián de un cese de fuego, al menos entre las principales actores armados enfrentados, como una masa crifica para la creación de coaliciones politicas susceptibies para convertirse en interlocutores claves para el apoyo y cocperación exterior. Segundo, apoyos logisticos y de transferencia de capacidades conducentes al diserio y organización de las fuerzas de seguridad tanto las de indole palicial como la miltar. Esto es fundamental para la pacificación del inferior del pais y una forma vital que basada en el despliegue ferntorial contribuya a la habiltación del poder juriscliccional del Estado y dote de recursos facultativos consubstanciales al ejercicio de la auforidad. Y tercero, el Estado debe ser regenerado en su inselicicionalidad especializada pera la economia nacional es bajo estas circunstancias en donde las iniciativas deben ir activando progresivamente una estrategia general basada en la construcción de Estado desde abajo, y que ya se ha explicado ampliamente..." Morales Perla, 2013 pag. 2031
\end{abstract}

\title{
III. Areas criticas de impacto.
}

Los niveles de impacto conciernen a los ámbitos de decisión y gestión, espacios dónde se procesan insumos y se generan dinámicas organizacionales. Por su parte se entenderá como área critica a toda aquella herramienta de gestion o expresión decisional cuyo flujo y desarrollo modifica entorno, moviliza objetivos, realiza metas, comunica actores y operarios, materializa productos, diseña procesos criticos y evalúa resultados.

Son cinco áreas criticas, que para los menesteres de situar la investigación cientifica de alcance politico- estratégico; es necesario presentar en este documento y ampliar algunas de sus caracteristicas. Todo ello concieme a los programas de un tanque de pensamiento en seguridad. La revista "Policia y Seguridad Pública", fiene como objetivos estratégicos:

1. Garantizar aportes cientificos de calidad para el fortalecimiento de las entidades de la comunidad de seguridad pública.

2. Generar propuestas avanzadas de alto impacto en las politicas públicas de seguridad.

3. Innovar con investigaciones de alto valor agregado en la educacion policial.

De estos se desprenden como áreas criticas para transformar, en primer lugar la cultura del planeamiento estatal, en segundo la ingenieria de la decisión pública, tercero la dinámica del asesoramiento estratégico, cuarto la propiciación de la cultura prospectiva y quinto la formación de 
analistas estratégicos. ${ }^{4}$ Describo a continuación algunos de los aspectos inherentes en las mismas, asi como sus dimensiones.

A. Cultura del planeamiento estatal: Debe emerger como práctica sistematica y generalizada en todo ámbito del Estado, pero aunque incluye las orientaciones para arios y quinquenios; deben ser permeadas por la cultura estrategica y elio conlleva a entoques transdisciplinarios y de largos plazos, contribuyendo a una mejor intersección entre los imperativos de seguridad y desarrollo. Significa que la cultura del planeamiento y los sistemas de trabajo que organiza para verificar y monitorear, se desarrollan cualitativamente para los efectos de pensar el futuro de las instituciones en cuanto a roles y misiones y su apoyo fundamental a los procesos criticos de un pais.

B. Ingenieria de la decisión pública: Conlleva al imperativo de radiografiar los sistemas bajo los cuales operan la toma de decisiones, los requerimientos de cuantificarla, evaluar los modelos, las formas y los mecanismos informales y discrecionales que median en la concepción, toma y ejecución de una decisión. Cómo se toman decisiones criticas, cuáles son las principales circunstancias de entorno que influyen, qué papel juegan los factores morales, racionales y emocionales; sobre todo cuando éstas se refieren a las del ámbito de seguridad. De quiénes y cómo se participa en las mismas. Lo anterior es clave para entender cómo se afecta la gobemabilidad, la conducción de politicas públicas y la generación de determinados resultados de gestión y desempeño.

C. Asesoramiento estratégico: Esta es un àrea critica, dadas las deficiencias que ya se han descrito. No existe escuela especializada que forme estos perfiles claves y en los mismos se carece de múltiples competencias y requisitos para tan sensibles menesteres. Salvo en paises desarrollados, el asesoramiento estratégico constituye una herramienta de rigor porque orienta el proceso de toma de decisiones, incide en el planeamiento y en los cursos de acción de los gobernantes. 5

El asesoramiento estratégico transfiere experiencia y si bien una masa critica de éste en el Estado, puede contar preliminarmente con una tuente de empirismo; se trata que el mismo se conduzca por una ruta de especialización muy particular como para hacer posibles umbrales de profesionalización necesarias en el sector seguridad.

D. Cultura prospectiva: La prospectiva como disciplina cientifica tiene escaso desarrollo en EI Salvador, por ello se ha articulado una linea estratégica de investigación que se denomina: "Estudios sobre el futuro y gestión de entomos complejos de seguridad". Al evolucionar el CINC hacia un tanque de pensamiento en seguridad, ésta linea se planteará como una de las más pujantes. Es muy especializada, requiere en muchos casos de software avanzado de simulación y sobre todo acceso y diálogo con funcionarios y expertos.

Al respecto éste suscrito apuntaba en la sección de "Carta del Editor", los siguientes problemas y carencias de este tipo de estudios en el pais:

\footnotetext{
" Vease discurso del coordinador principal del CNC.ANSP, pronunciado en la presentacion publica del sexto número de la revista "Policla y Seguridad Publica" sobre la temśtica "Prospectiva de Seguridod año 5 vol, 1 p.445

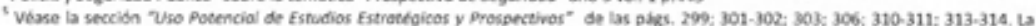
sección "Conclusiones" p. 316-318 y "Mecomendociones Cutrotelgicas y Desafios futuros" p. 318-323. En Morales Pela, Juan Carlos. "Los Etudios Eatretigicos en El Solvador. Su potencial paro el desorrollo de centros prospectives de pensamiento avancado pera lo seguridad nocionaly la defenso". Mho 4, vol. 2, 2015.
} 
'Lo que si ha existido en El Salvador es predicoion thenica que no es prospectiva tal y como se le entende; estos reportes han sevido para explicar el comportamiento y las proyecciones econdmicas de crecimiento o en áreas tin distintas como la demografa como de alto interes por lo fomo do como sus dinámicas y fendencias inciden en el planeamiento general del Estado.

A il antenor se suman otras caracteristicas que hen estodo inherentes en la producoidn intelectual nucional, entre estas:

1. Predominio de estudios sociolbgicos cribicos de la realided, ditundidos y amplamente aceptados; pero desprovistos de vanuoves thunbles, con carencie de escenarios estradtgicos importantes

3. Ausencia do una culura de la prospecfive en la fomacion profesional y muy evidente en la producción infelectual del pais. Nade insgins el fturo a largo plazo, prevalece la firmula del que hay que hacer, pero no el como hacerlo y el hacia ¿donde?

4. Las imitados antecedentes de producción inteloctual con el uso de tecricas cuantiativas especializadas de la prospectiva: oue sin ambargo no son las inicas para disehier y modolar fitunbles, han sido aplicados al ámbito de ia polisica y la gobemabildad pero presentando dos aspectos negstvos: escasez de proyeccón y valoración estrategica de probabisdades e incapacidad para imaginar futuros con umbrales de incertidumbre, fipologla de entomos, estimaciones de sempo entre otros. El esfuerzo culmina con un formuto gis. cargado de reflexion socio-poltica: muy fel a la usanza acadenica del mundo de las ciencias socivies, on of que desgrasiodamonte hay que decir y elaborar mucho pera bo que puede estar contenido en une pstabra o idea'6 (Morales Poha, 2014 , Q.17).

Pensar en el futuro del Estado es de orden fundamental, imaginar los escenarios estratégicos, recrearlos, explorarlos, estimar politicas en tiempo presente, en ambientes que permiten modificar variables; son todos en su conjunto recursos avanzados para la gestión de la seguridad, y por ello vital propiciarlo como cultura, como práctica, como intersección importante con los programas de investigación en el àmbito público.

\section{E. Formación de Analistas Estratégicos:}

Existe necesidad de analistas en el pais, más aún en el vasto campo de la seguridad y sus distintas áreas especializadas. Al respecto se sabe que no hay programas para formarlos, que predomina la cultura del empirismo y que las experticias se desarrollan en la práctica profesional y en el monitoreo cotidiano de problemas y gestion de amenazas. En El Salvador se requieren 'perfiles de prospectivistas, especialistas de la estrategia y la producción de inteligencia. El arte de asesorar, va más allá de la cultura del power point y la literatura gris reservada. Implica tareas que incluye la producción de informes y otros documentos de gran valia, pero que los mismos se inscriban e impacten en los cursos de acción de las jefaturas y jerarquias que se ubican en las lineas criticas de la decisión y la intervención en materia de seguridad.

\footnotetext{
"Véase la sección "Carta del Editor" aparecida en el sexto número de la revista sobre "Prospectiva de Seguridad". Año 4, vol. 2, p.16-18, 2014
} 


\section{Centro de Investigación Cientifica CINC 2015}

IV. Evaluación de Politicas Editoriales y de Gestión Cientifica.

(A partir de la experiencia de la publicación élite "Policia y Seguridad Pública")

\section{1. Área ética:}

Politica:

1.1 Se garantizará la protección de datos personales de colaboradores académicos, pares evaluadores u otro personal involucrado en la gestión editorial.

Evaluación:

1.1 Proceso anónimo y confidencial. Constituye una práctica desde el primer volumen publicado. Politica:

1.2 Se promoverá la búsqueda de la verdad, la justicia social y el bien común. Evaluación:

1.2 Imperativos y esfuerzos orientados a prevenir de toda influencia negativa, ideologica y partidaria; asi también de consideraciones económicas y politicas interesadas y no sustentadas. Al respecto se han habilitado filtros, formatos de control y arbitrajes.

Politica:

1.3 Se apreciará en el más amplio sentido todo producto académico apoyado en una sólida evidencia cientifica que contraste intereses creados.

Evaluación:

1.3 Regulado con formatos y otros instrumentos de evaluación y verificación en los arbitrajes y rutas de mejora, términos de referencia (TDR) y otros.

Politica:

1.4 Se inhabilitará en forma temporal o permanente a todos aquellos académicos a quienes se demuestre prácticas sistemáticas de plagio u otros vicios académicos fundamentales.

Evaluación:

1.4 Orientados a generar estructuraciones importantes, propuestas alternativas de mejora y actualización. Para ello se ha oficializado y operativizado la inclusión de una "Declaratoria de arbitraje' que orienta a pares evaluadores y autores; además de una "Doclaratoria de originalidad", que compromete a los participantes y les previene de los trabajos con publicaciones previas o postulaciones simultáneas, constituye un atestado, fipo declaración jurada; en definitiva un instrumento que transparenta el proceso.

Politica:

1.5 Se protegerán atestados de evaluación y dictámenes que formulen los pares evaluadores durante y después de la fase de arbitraje.

\section{Evaluación:}

1.6 Ha constituido una práctica desde el primer volumen publicado. En algunas ocasiones y ante la solicitud del autor que ha recibido dictámenes desfavorables en torno a su articulo; el grupo editor ha tenido a bien compartir el expediente completo, para satisfacer aspectos de ampliación y contundencia de un proceso de evaluación. Ello se ha hecho resguardando las señas personales de autores y evaluadores.

\section{2. Área de producción intelectual y conocimiento: Politica:}

2.1 Se instituirán y fortalecerán periódicamente incentivos materiales, económicos y morales para la comunidad de investigadores en su carácter de colaboradores académicos, evaluadores u otros participantes intelectuales activos.

Evaluación: 


\section{Centro de Investigación Científica CINC 2015}

2.1 Via incremento de fondo de investigación, presupuesto que ha permito desarrollar crecientes proyectos de consultoria. En el ámbito material se retribuye con la entrega estipulada de ejemplares a pares y autores; $y$ siendo en el ámbito de incentivos morales, el reconocimiento de créditos académicos y curriculares en las diversas ediciones publicadas.

Politica:

2.2 Se medirá anualmente el impacto social e institucional de los trabajos publicados y se certificará a los investigadores y autores de los mismos.

\section{Evaluación:}

2.2 La medición y evaluación de impactos requiere de inversión estratégica sostenida, para arribar al diseño de instrumentos especializados y registro de experiencias. Sin embargo el cumplimiento de estas politicas se avanzan en la actualidad bajo la modalidad de dos formatos: En primer lugar a través del recurso de un estudio o investigación sobre la materia y en segundo lugar mediante la inclusión de informes estratégicos con sus respectivas estadisticas. En definitiva debe potenciarse hacia un tercer quinquenio.

\section{3. Área de desarrollo cientifico y sector productivo:} Politica:

3.1 Fomentara investigaciones prácticas de factibilidad financiera y de alta contribución substancial a los programas de investigación vigentes.

\section{Evaluación:}

3.1 Se han desarrollado tres tipos de incentivos los económicos, materiales y morales.

En las primeras ha mediado financiamiento de estudios, en el segundo entrega de un determinado número de revistas a autores y pares; $\mathrm{y}$ en tercero reconocimiento de créditos académicos en cada publicación en sus siete distintas categorias: (1) autor, (2) par evaluador, (3) miembro del Consejo Cientifico Editorial; (4) Colaborador IV (Elabora sinopsis de cine y documentales); (5) Colaborador III (Elabora sinopsis de documentos web); (6) Colaborador II (Elabora sinopsis de libros) y Colaborador I (Elabora recensiones de libro). Además se ha recurrido a la búsqueda diversificada de expertos e investigadores jóvenes emergentes. Las inversiones en materia de investigación han fluctuado en tarifas de entre $\$ 600.00$ dólares americanos, la minima; incluyendo otras por $\$ 2,500.00 ; \$ 4,000.00$; hasta alcanzar montos por $\$ 7,000.00$ dólares americanos.

Politica:

3.2 Estimulará investigaciones con un alto uso potencial para el diseño de politicas públicas de seguridad o de aporte cualitativo a los tomadores de decisiones.

\section{Evaluación:}

3.2 Ha desarrollado una plataforma de "Estudios Estratégicos de Seguridad" e incorporado las lineas de investigación hacia ese rubro especializado. 1- "Manejo y prevención de crisis y conflictos sociales"; 2. "Estudios sobre el futuro y gestion de entomos complejos de seguridad" y 3- "Diseño inteligente de politicas públicas de seguridad". En adelante podrán cuantificarse el número de estudios e investigaciones por año y que abonan a cada linea estratégica.

\section{Politica:}

3.3 Propondrá alianzas estratégicas con otras revistas académicas y cientificas del mismo rubro o materia de especialización; u otros mecanismos de colaboración que se estimen pertinentes y que gocen de estándares internacionales de reconocimiento universal.

\section{Evaluación:}

3.3 El impulso de esta politica, se sujeta al avance de mayores niveles de indización internacional en bases académicas. Por ello existe un empeño fuerte actual para lograr el ingreso a otros 
reservorios académicos en linea. Actualmente se ha logrado el acceso a siete sitios especializados y se proyecta igual cantidad de nuevas filiaciones.

Politica:

3.4 Diseñará indices de medición y evaluación de desempeño, calidad e impacto cientifico.

Evaluación:

3.4 En materia de gestión cientifica y en los imperativos de la investigación en seguridad, se requiere una muestra minima de al menos 10 ediciones.

Pero se declaró como politica desde el principio, porque un CINC o CINCEES debe evolucionar en esa dirección. A la fecha se han determinado niveles de impacto como escenarios fundamentales, y de los cuáles se hace un esbozo en el presente documento. Y se ha mudado el Formato F- 1 a un instrumento de recolección estadistica de rastreo de indicadores cientificas en los estudios publicados. Esto podrá generar informes estratégicos y otros instrumentos factibles.

\section{4. Área de dífusión del conocimiento:}

\section{Política:}

4.1 Publicará una edición especial que recoja y organice indices de trabajos académicos, colaboradores intelectuales y sus filiaciones profesionales e institucionales y posteriormente de evaluadores, una vez se cumpla la condición indispensable de consolidación de bases de datos. Evaluación:

4.1 El cumplimiento de esta politica, tendrá como marco de referencia los proyectos de publicación número (8) sobre "Inteligencia Estratégica" y en el (9) "Control social y territorial".

Politica:

4.2 Publicará o incluirá en sus números editados, trabajos de autores que publican en otras revistas académicas y con las cuales se sostengan convenios en materia de cooperación cientifica.

Evaluación:

4.2 Estas iniciativas aún no fructifican. Se trabaja en un concepto de alianza con revistas especializadas del mismo rubro de publicación o similares; y se apuesta a esfuerzos de trayectoria en Colombia, Catalunya y Chile. Esto deberá ser nomado y regulado por un instrumento de convenio entre grupos editores.

Politica:

4.3 Promoverá el ingreso de esta publicación a bases de datos indizadas para los efectos de una mejor visibilización y relacionamiento de nuestros autores con la comunidad académica internacional.

Evaluación:

4.3 A la fecha la publicación "Policia y Seguridad Pública" ha ingresado y sido indizada en siete bases de datos académicas internacionales, las cuales son: Vlex, Latindex, Lamjol, Google Académico, Dialnet y Clase. Las cuales pertenecen a paises tan distintos como México, Estados Unidos de América, España y el Reino Unido.

Actualmente se avanzan gestiones para tramitar el ingreso a otras bases, en algunas de las cuales, la publicación debe pasar un periodo de evaluación de su candidatura, revisión de sus parámetros editoriales e incluso su clasificación en una escala de tipologia de revistas.

\section{Area de investigación y docencia:} Politica:

5.1 Concertará con equipos de investigadores de otras revistas académicas o comunidades cientificas; esfuerzos conjuntos de investigación en su carácter transdisciplinario e 
interinstitucional. Favoreciendo las modalidades basadas en el concepto de asocio públicopúblico.

\section{Evaluación:}

5.1 Se ha cumplido y se encuentra en plena expansion. El grupo editor ha sido pionero en inaugurar una cultura basada en una modalidad de asocio público-público. Esto ha permitido generar estudios transdisciplinarios de campos tan diversos como Psicologia, Antropologia, Estudios Estratégicos, Prospectiva, Inteligencia, Ciencias Sociales, Economia, Sociologia, Ciencias Juridicas, Criminologia, Historia, entre otros. Convocando en el proceso a entidades de investigación del sector Defensa, Salud Pública, Economia, Sistema financiero, Educación y naturalmente Seguridad Pública.

Politica:

5.2 Integrará al menos dos docentes o funcionarios de la comunidad educativa de la ANSP para el aporte sistemático de productos de investigación para cada número de la revista institucional en plena coincidencia de los programas de investigación vigentes.

\section{Evaluación:}

5.2 Esto no ha sido posible por las caracteristicas inherentes en la comunidad docente de la ANSP. las experiencias fracasadas del pasado y la falta de funcionalidad y operatividad de antiguas instancias concebidas en el proyecto original CINC como el Consejo Consultivo, en donde hubo un modelo de representaciôn de departamentos subordinados a la División de Estudios.

Modelo que fue derogado y modficado posteriormente, a raiz de los pobres resultados y los imperativos de desarrollo en los que se orientaba el CINC.

Sin embargo ha operado la modalidad de reinvindicar los mejores estudios de los distintos cursos de ascenso. Dinámica que ha permitido la inclusión de un estudio de miembros de las Policia Nacional Civil (PNC) en el número (6) sobre "Prospectiva de Seguridad", la inclusión de otro en el número (7) sobre "Pandillas y Extorsiones" y se estima un umbral favorable para la inclusion de otros estudios en los números (8) "Inteligencia Estratégica y (9) "Control Social y Territorial".

Politica:

5.3 Garantizará la libertad académica de ideas, condición que se cenirá a la fiel observancia de los formatos cientificos u otros propios del quehacer investigativo.

\section{Evaluación:}

5.3 Se viene cumpliendo satisfactoriamente, el grupo editor ha hecho extensivas las convocatorias. ha cerrado acuerdos de colaboración y propiciado proyectos de consultoria con distintos académicos y expertos sin distingos ideológicos, politicos o partidiarios. Los atestados de estos resultados constan en las estadisticas presentadas en este documento y en el detalle de las filiaciones de todos los participantes que han concursado en los distintos números editados.

\section{Patrimonio y legado del Centro de Investigación Cientifica (CINC)}

1. Cultura del Arbitraje: Con el surgimiento de la revista "Policia y Seguridad Publica", que se basa en el formato de un journal de seguridad; se inauguró la cultura del arbitraje a nivel de pais, constituyó la primera del sector público en organizarlo, sistematizarlo y establecerlo como práctica; porque constituye un aspecto evaluado como parámetro de gestión cientifica y editorial, un requisito indispensable para la indización en bases de datos academicas intemacionales. El arbitraje es un proceso adoptado en el Centro de Investigación Científica desde la publicación del primer volumen en diciembre de 2011. Este parámetro desarrollado en los años subsecuentes, permitio liderar a nivel nacional en el mundo de las revistas cientificas de diversas entidades privadas y universidades. Pronto fue adoptado por otros consejos editores y 
Propuesta de conversión del Centro de Investigación Científica (CINC) en Centro de Investigación Científica...

consiguientemente imitadas las buenas prácticas con las que "Policia y Seguridad Pública" ha sido vanguardia en el pais y la región.

El arbitraje comprende tres procesos criticos. Estos conciemen a un primero que se denomina "Recepción y verificación preliminar", que incluye el acceso al documento o estudio en su carácter candidato, para ser revisado por el grupo editor, el cual determina si reúne los aspectos básicos de rigor para proceder a una conformación de ternas de evaluadores. La segunda etapa compete a la "realización de una ronda de consultas" entre las comunidades de especialistas, para los efectos de conformar ternas de evaluadores y en la que se debe contar con el perfil requerido a nivel profesional y académico, según instrucciones que se hacen circular. Una vez las ternas se conforman, se les remiten atestados de rigor que conciemen a la invitación formal, el trabajo a evaluar y los formatos de gestión para la práctica del arbitraje entre estos el F-1/ Formato de identificación de información de relevancia cientifica; F-3/ Formato de redacción de dictamen y F-6/ Formato de manejo de borrador electrónico comentado. En esta etapa se estipula fechas de entrega y devolución, es un proceso confidencial y anónimo y está garantizado por la politica ética que se declara en la revista.

Por su parte la tercera etapa conlleva a la sistematización de resultados de dictámenes y su devolución a los autores para los efectos de implementación obligatoria de las rutas de mejora. El arbitraje pretende entre otros objetivos: 1- Detectar plagios; 2- Identificar vicios académicos y malas prácticas; 3- Proponer aspectos de rigor a solventar; 4- Verificar impactos importantes 0 potenciales; 5- Prevenir de las postulaciones simultáneas; 6- Identificar aspectos de relevancia cientifica; 7. Volver asequible y potable un estudio con temáticas sensibles; 8- Blindarlo de la publicación sin filtros y monitoreo cientificos; 9- Robustecerlo con apreciación y argumentos calificados de alto valor agregado; 10. Situarlo para la discusión academica y pública; 11 . Evaluarlo por su contribución a un sector o sistema de trabajo en particular; 12- Analizar su aporte substancial al estudio de un problema 0 al avance de la disciplina cientifica en la que se inscribe; entre otros.

A continuaciön algunas estadisticas, de la observación y práctica del arbitraje en las últimas tres revistas de "Policia y Seguridad Pública" en sus distintas opciones de resultados del proceso de evaluación: Los favorables, los favorables condicionados y rechazados.

\section{Cuadro 1}

Muestreo estadistico sobre dictámenes de los últimos tres números

\begin{tabular}{|l|c|c|c|}
\hline Número de Revista & \multicolumn{3}{|c|}{ Tipo de Dictamen } \\
\hline Opciones & Favorables & $\begin{array}{c}\text { Favorables } \\
\text { condicionados }\end{array}$ & Rechazados \\
\hline Revista \# 5 & 1 & 23 & 5 \\
\hline Revista \# 6 & - & 19 & 3 \\
\hline Revista \#7 & - & 16 & 5 \\
\hline
\end{tabular}

Nota importante: En los casos de la revista \# 4 "Población Reclusa y Violencia Carcelaria" y la \# 3 "Narcotráfico y Lavado de Activas"; también se cuentan con estadisticas disponibles. No se incluyeron en el cuadro, porque el propósito didáctico del mismo es ilustrar la tendencia de los últimos dos anios. En el caso de las estadisticas de los números \#1 y \#2; el archivo y su levantamiento se encuentran en proceso de sistematización, pues implica la recuperación de 
dictámenes en su formato de adjunto remitidos a las distintas cuentas de correo electrónico del Centro de Investigación Cientifica.

Cuadro (2)

Procedencia de los académicos participantes por número publicado de la revista "Policia y Seguridad Pública"

\begin{tabular}{|c|c|c|c|c|c|c|c|c|c|}
\hline Autores & $\begin{array}{c}\text { Rer. } \\
\text { (1) } \\
2011\end{array}$ & $\begin{array}{l}\text { Rev. } \\
\text { (2) } \\
2012\end{array}$ & $\begin{array}{l}\text { Rev. } \\
\text { (3) } \\
2013\end{array}$ & $\begin{array}{c}\text { Rev. } \\
\text { (4) } \\
2013\end{array}$ & $\begin{array}{c}\text { Rev. } \\
\text { (5) } \\
2014\end{array}$ & $\begin{array}{c}\text { Rev. } \\
\text { (6) } \\
2014\end{array}$ & $\begin{array}{c}\text { Rev. } \\
(7) \\
2014\end{array}$ & Total & Porcentaje \\
\hline Institucionales" & 2 & 3 & 1 & 1 & 2 & 1 & 1 & 11 & $19 \%$ \\
\hline Nacionales" & 3 & 2 & 3 & 2 & 4 & 4 & 3 & 21 & $36 \%$ \\
\hline Internacionales":- & 6 & 4 & 1 & 6 & 3 & 3 & 4 & 27 & $46 \%$ \\
\hline Total & 11 & 9 & 5 & 9 & 9 & 8 & 8 & 59 & $100 \%$ \\
\hline
\end{tabular}

"Institucionales: Centio de Invesfgación Cientfica, Division de Estudios, Depatamento de Senvicios Estatales y Privados de Seguidad.

"Nacionales: Universidad de El Salvador, Instuto de Cencias, Tecrologia e Innovación (UFG), Organo Judiciat Insthato Naxional de la Juventud (INUUVE), Colegio de Allos Estudios Estrategicos (CAEE). Academia Salvadoveha de la Fistoria. Ministerio de Trabajo y Previsión Sociat; Acadenia de Historia Miltar Salvadorefia, Circulo de Escritones Miltares; Centro Nacional de investigaciones en Ciencias Sociales y Humanidades (CENICSH); Faculad de Clencias y Humanidades (UES); Escuela de Clencias Sociales (UES) Policla Naciona Cid (PNC); Insthto Especializado de Educacion Superior de Prolesionales de la Salud de El Savador (IEPROES)

-"'internacionales: Argentna, Italia, Estados Unidos de America, Espaha, Colombia, Francia, (UNLPEC) (CIPC). Centro Regional de las Naciones Unidas para la Paz el Desarme y el Desarnollo en Ametica Lajina y el Caribe, Brasil (Insthtoto de Invessgasion Economica) y Aplicadal. Mevico; Coiombia (Universidad de Colombia); Espena (Policia de la Generartitat -Mossos de 'Squadra, Secretaria de Gobierno Santa Fe de Bogota, Universidad de Murcia, Centro de Estudios Junicicos y Formacion Especialzada (CE.JFE). Uriversidad Ramon Uult, Departamento de Justicia de la Gererailat de Catalunya, Insthito de ciencias Forenses y de la Segunidad (Universidas Aulchoma de Madid. Espana); Nasional Deferse University (NOU. Venezuela?

Nota importante: Este cuadro fue incluido en un estudio publicado en la revista \# 6 sobre "Prospectiva de Seguridad", pero en su momento el muestreo de analisis abarcó hasta la revista \# 5. He aqui la estadistica actualizada hasta el recientemente publicado \#7 sobre "Pandillas y Extorsiones". Lo destacable aca es que la participación de los tres grupos de académicos claves y sus filiaciones, se encuentra razonablemente proporcionales y equilibrados tanto en la estadistica global como en la particular de cada número editado.

\section{Cuadro (3)}

Procedencia de los pares evaluadores y árbitros de investigaciones y estudios por número publicado de la revista "Policia y Seguridad Pública"

\begin{tabular}{|c|c|c|c|c|c|c|c|c|c|}
\hline Evaluadores & $\begin{array}{c}\text { Rev. } \\
\text { (1) } \\
2011\end{array}$ & $\begin{array}{c}\text { Rev. } \\
(2) \\
2012\end{array}$ & $\begin{array}{c}\text { Rev. } \\
(3) \\
2013\end{array}$ & $\begin{array}{c}\text { Rev. } \\
\text { (4) } \\
2013\end{array}$ & $\begin{array}{l}\text { Rev. } \\
\text { (5) } \\
2014\end{array}$ & $\begin{array}{c}\text { Rev. } \\
(6) \\
2014\end{array}$ & $\begin{array}{c}\text { Rev. } \\
\text { (7) } \\
2014\end{array}$ & Total & Porcentaje \\
\hline Insthucionales" & 2 & 2 & 0 & 3 & 3 & 9 & 4 & 23 & $12 \%$ \\
\hline Nacionales"* & 2 & 7 & 6 & 6 & 11 & 4 & 5 & 41 & $37 \%$ \\
\hline Intemacionales ${ }^{\prime \prime \prime}$ & 10 & 5 & 8 & 11 & 10 & 6 & 8 & 58 & $51 \%$ \\
\hline Total & 14 & 14 & 14 & 20 & 24 & 19 & 17 & 122 & $100 \%$ \\
\hline Porcentaje & $16 \%$ & $16 \%$ & $16 \%$ & $23 \%$ & $28 \%$ & $22 \%$ & $20 \%$ & $100 \%$ & $=$ \\
\hline
\end{tabular}

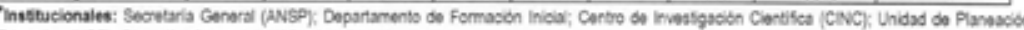

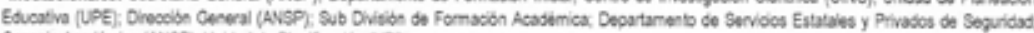
Consejo Academios (ANSP), Unidad de Planílcadón (UP).

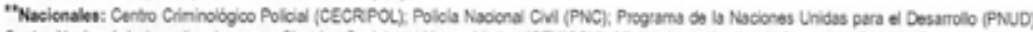
Cento Nadional de investgaciones en Cencias Sociales y Humanicades (CBVICSH); Ministero of Justica y Seguridad Publica. Departamento de

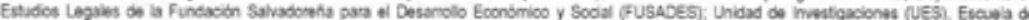

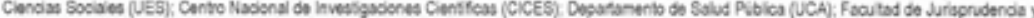

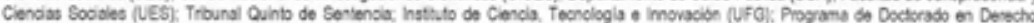

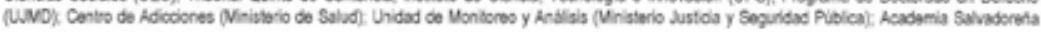


Propuesta de conversión del Centro de Investigación Científica (CINC) en Centro de Investigación Científica...

\section{Centro de Investigación Científica CINC 2015}

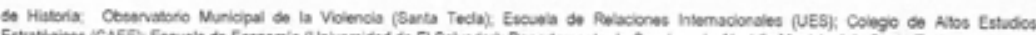

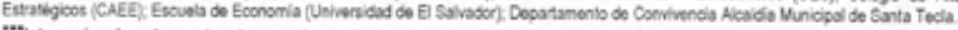

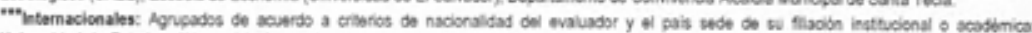
Universidad de Potsdam (Alemana); Causa Comin (Nexico); Univaridad de Barcelona (Espalla); Centes Regional de las Nadones Unidas para la

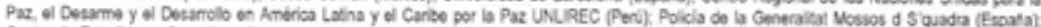

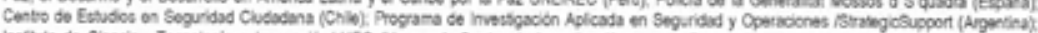

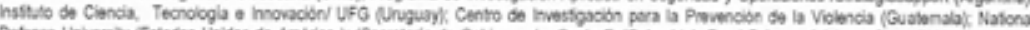

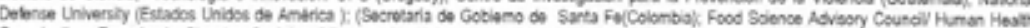

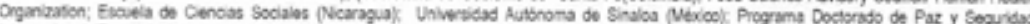

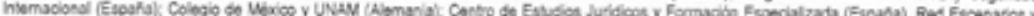

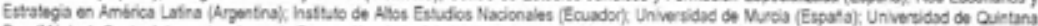

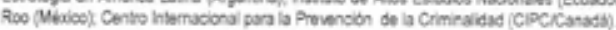

Nota importante: Este cuadro fue incluido en un estudio publicado en la revista \# 6 sobre "Prospectiva de Seguridad", pero en su momento el muestreo de analisis abarcó hasta la revista \# 5 He aqui la estadistica actualizada hasta el recientemente publicado \# 7 sobre "Pandillas y Extorsiones". Lo destacable acá es que la participación de los tres grupos de académicos claves y sus filiaciones, se encuentra razonablemente proporcionales y equilibrados tanto en la estadistica global como en la particular de cada número editado.

Con ello se cumple con los tercios requeridos para cada grupo y que constituye un parámetro de gestión editorial y cientifica.

\begin{tabular}{|c|c|c|c|}
\hline Nümero revista y edicion & $\begin{array}{l}\text { Nümero de articulos } \\
\text { cientificos y estudios } \\
\text { entratiojicos }\end{array}$ & $\begin{array}{l}\text { Promedio de paginas } \\
\text { de artculos por } \\
\text { ediclibe" }\end{array}$ & Poccentaje \\
\hline A60 1, Vol, 1, Jula-Didentibe 2011 & 7 & $280 / 350$ & $80 \%$ \\
\hline Ato 2, Vol. 1, Envoro-Junio 2012 & 8 & $255 / 350$ & $73 \%$ \\
\hline $\begin{array}{l}\text { Alo 2 Vo. 2, Julo 2012-Didembre } \\
2013\end{array}$ & 5 & $205 / 333$ & $62 \%$ \\
\hline ANo 3, Vol. 1, Vayo-Oction 2013 & 6 & $250 / 399$ & $63 \%$ \\
\hline $\begin{array}{l}\text { A10 4, Vob. 1, Novientere } 2013 \text { - Junio } \\
2014\end{array}$ & 8 & $302 / 430$ & $70 \%$ \\
\hline Ano 4, Va. 2. Julo-Dodemben 2014 & 8 & $335 / 456$ & $74 \%$ \\
\hline Alo 5, Vos. 1, Entos Junio 2015 & 8 & $393 / 484$ & $81 \%$ \\
\hline Totales & 50 & $2,020 / 2,802$ & $72 \%$ \\
\hline
\end{tabular}

-Se especifca la cartidad de contenidos etectives en materia de estadics e imvestgaciones sobre el total de paginas de cada volumen publicado.

Nota importante: El $72 \%$ en promedio de los contenidos de cada número editado de la revista "Policia y Seguridad Pública"; lo ha constituido estudios e investigaciones originales e inéditos. congruentes con cada convocatoria y fiel a la agenda de investigación vigente. Destaca el 
primer volumen de 2011, cuyo porcentaje de contenidos de investigación alcanzó el $80 \%$ y el \# 6 sobre "Prospectivas de Seguridad" con $81 \%$.

El parámetro estipulado internacionalmente sobre este asunto es de $60 \%$ como minimo; en el CINC-ANSP se ha cumplido sobradamente.

\section{Divulgación nacional e internacional:}

Todo conocimiento sino se divulga fracasa, si a esto se agrega que no se distribuye ni circula entre los públicos meta estratégicos; se comprometen entonces en el largo plazo los distintos impactos que ya se abordaron en sección anterior.

Desde el surgimiento de la revista \#1 que desarrolló la temática sobre "Policia Comunitaria y Prevención de Violencia", se ha distribuido a más de 150 destinos en el exterior, que incluyen los cinco continentes, abarcando tanques de pensamiento en materia de politica exterior, seguridad, defensa, y economia; universidades; centros de investigación; institutos especializados; redes de expertos; academias de policias; policias extranjeras; cónclaves académicos; foros internacionales de funcionarios; escuelas especializadas; entre otros.

Asi también para la escogitación y elaboración de una nómina de sitios relevantes para tareas de distribución de ejemplares, se ha considerado varios criterios entre los que destacan: 1- Tipo de institución; 2- Area critica de desempeño de la institución; 3- Oportunidad para el establecimiento de alianzas estratégicas; 4- Compromiso con pares evaluadores para reconocimiento (remisión de atestados para reconocimiento de créditos a pares evaluadores); 5 . Remisión a miembros del Consejo Cientifico Editorial por labores de asesoramiento y 6 Oportunidad de acuerdos de cooperación académica (individual o colectiva). A continuación, el detalle completo de todas estas instituciones y entidades:

\section{Destinos de envio de la revista: Paises y continentes INSTITUCIONES NACIONALES:}

(148 entidades en total, con sede en el pais: Incluye 30 sedes diplomáticas; 12 universidades y 8 institutos de educación superior)

Embajada de Canadá, Embajada de los Estados Unidos de América , Embajada de México, Embajador de Belice, Embajada de Guatemala, Embajador de Honduras, Embajada de Nicaragua, Embajada de Costa Rica, Embajada de Panamá, Embajada de Colombia, Embajada de Ecuador, Embajada de Venezuela, Embajada de Peru, Embajada de Unuguay, Embajador de Chile, Embajada de Brasil, Embajada de Argentina, Embajada de Cuba, Embajador de República Dominicana, Embajada de España, Embajada de Italia, Embajada de Francia, Embajada de Alemania, Consulado de Suiza, Embajada de Israel, Embajada Estado de Qatar, Embajada de Malta, Embajada de Japón, Embajador de China, Embajada de Corea Universidad de El Salvador (UES), Universidad Centroamericana "José Simeón Cañas" (UCA). Ministerio de Salud Pública y Asistencia Social (MSPAS), Universidad Tecnológica de El Salvador (UTEC), Universidad Don Bosco (UDB), Universidad Evangélica de El Salvador (UEES), Universidad José Matias Delgado (UJMD), Instituto Tecnológico Centroamericano (ITCA), Universidad Francisco Gavidia (UFG) Universidad Católica de El Salvador, Universidad de Sonsonate, Colegio de Altos Estudios Centroamericanos, Universidad Modular Abierta (UMA), Instituto Nacional de Salud, Universidad Oscar Amulfo Romero, Universidad Luterana Salvadoreña, Escuela Superior de Economia y Negocios, Comando de Doctrina Militar (CODEM) Colegio de Altos Estudios Estratégicos (CAEE), Instituto de Educación Superior para Profesionales de la Salud de El Salvador (IEPROES), Instituto Superior de Economia y Administración de Empresas ISEADE/FEPADE. 
Propuesta de conversión del Centro de Investigación Científica (CINC) en Centro de Investigación Científica...

(Incluye 32 ministerios y autónomas del sector público; 2 centros de investigación y 15 alcaldias municipales)

Tribunal de Ética Gubernamental, Sub Secretaria de Transparencia, Instituto de Acceso a la Información Pública, Secretaria de Participación Ciudadana y Transparencia Anticorrupción (Antes la Subsecretaria de Transparencia Anticorrupción), Secretaria de Cultura, Centro Nacional de Investigaciones y Ciencias Sociales y Humanidades CENISCH-MINED, Instituto Salvadoreño del Seguro Social, SIBASI La Libertad, Ministerio de Justicia y Seguridad Pública, Ministerio de Educación, Ministerio de Medio Ambiente y Recursos Naturales, CORSATUR, Asamblea Legislativa, CONACYT, Consejo Nacional de la Niñez y la Adolescencia, Ministerio de Relaciones Exteriores, Fiscalia General de la República, Ministerio de Gobernación, CONJUVE, Corte Suprema de Justicia, Procuraduria General de la República, Ministerio de Hacienda, Ministerio de la Defensa Nacional, Administración Nacional de Acueductos y Alcantarillados (ANDA), Loteria Nacional de Beneficencia LNB, Fondo Social para la Vivienda, Ministerio de Turismo, Ministerio de Economia, Ministerio de Obras Públicas, Procuraduria para la Defensa de los Derechos Humanos, Corte de Cuentas de la República, Superintendencia del Sistema Financiero, Ministerio de Trabajo, Instituto Salvadoreño de Transformación Agraria (ISTA). Comisión Ejecutiva Portuaria Autónoma (CEPA), Fondo de Inversión Social para el Desarrollo Local (FISDL), Consejo Nacional de la Energia (CNE), Dirección General de Centros Penales, Casa Presidencial (CAPRES), Policia Nacional Civil y sus diferentes unidades, Comisiön Nacional Antidrogas, Observatorio Municipal de la Violencia Antiguo Cuscatlán, Observatorio Municipal de la Violencia San Salvador, Observatorio Municipal de la Violencia Santa Tecla, Alcaldias Municipales del Gran San Salvador, Biblioteca Nacional Dr. Francisco Gavidia (UFG), Consejo de Alcaldes del Área Metropolitana de San Salvador (COAMSS/OPAMSS), Canal 10, Instituto de Educación Superior para la Formación Diplomática (IEESFORD), Consejo Académico de la Academia Nacional de Seguridad Pública (ANSP), Instituto de Medicina Legal, INTERPOL.

\section{INSTITUCIONES PRIVADAS}

Fundación de Estudios para la Aplicación del Derecho (FESPAD), ICITAD, Pro Búsqueda, INFORNEX, Centro de Desarrollo de Liderazgo (CEDELID), Fundación Nacional para el Desarrollo (FUNDE), Facultad Latinoamericana de Ciencias Sociales (FLACSO), Academia Salvadoreña de Historia, Editorial Santillana, Comisión Provincial por la Memoria, VM Quality S.A. de C.V., CCPVS, Fundación Construir, Fundación Alternativa, Talleres Gráficos UCA, Flor de Piedra, Parroquia Nuestra Señora de Los Ángeles, Asociación Nacional de la Empresa Privada (ANEP), Fundación INELSA, Grupo Golàn.

ORGANISMOS INTERNACIONALES ACREDITADOS EN EL PAIS

JICA, RTI, Banco Mundial, OEA, KOICA, GIZ, Delegación de la Unión Europea, SICA, ILEA, Programa INL USA Embassy, PNUD, FIECA

MEDIOS DE COMUNICACION

Radio KL, Canal 12, La Prensa Gráfica, El Diario de Hoy, Diario El Mundo, La Chèvere, Diario

Colatino, El Noticiero, Radio Cuscattan, Radio Maya Visión

PARTIDOS POLITICOS DE TODAS LAS FRACCIONES Y PARLACEN 


\section{INSTITUCIONES INTERNACIONALES:}

(190 entidades en total)

ESPAÑA (31 entidades): Universidad de Barcelona, CLAE-España, CEESC, Instituto de Estudios para la Paz y la Cooperación, Revista América Latina Hoy, Instituto de Iberoamérica, Universidad de Salamanca, Instituto Universitario de Estudios de la Ciencia y la Tecnologia, Residencia Universitaria "Colegio de Oviodo" Universidad de Salamanca", Revista CIDOB, International Affairs, Centro Superior de Estudios de la Defensa Nacional (CEDESEN), Instituto Español de Estudios Estratégicos, Instituto Juan Velásquez de Velasco, Instituto Universitario General Gutierrez Mellado (UNED), Real Instituto Elcano, Revista Migraciones Forzadas. Instituto Universitario de Desarrollo Social y Paz-Universidad de Alicante, Instituto de Altos Estudios Europeos, Universidad Autónoma de Madrid, Facultad de Filosofia y Letras Departamentos de Estudios Árabes e Islámicos y Estudios Orientales, Escuela Diplomática del Ministerio de Asuntos Exteriores y de Cooperación de España, Instituto Universitario de la Paz y los Conflictos, Centro de Documentación Cientifica, Universidad de Granada Departamento Psicologia Social y Metodologia de las Ciencias del Comportamiento, Responsable de Projectes i Organizació Direcció General d'Administracio de Seguretat Departamentd'Interior, Director Instituto Vasco de Criminologia, Maestria en Analista de Inteligencia Universidad Rey Juan Carlos, Facultad de Educación Universidad de Murcia, Área Central de Análisis, Comisaria General De Informaciön Complex Central de la PG-ME, Institut de Seguretat Pública de Catalunya

ALEMANIA (14 entidades): Transparency International, Univertităt Passau, Instituto de Estudios Latinoamericanos, Ibero-Amerikanishches Institut Preubischer Kulturbesitz, Institut für Internationale Konfikttorschung, GIGA German Institute of Global and Area Studies LeibnizInstitut für Globale und Regionale Studien Universidad de Franckfort, Universidad de Münster, Instituto Max Plack, Deutscher Akademischer Austausch Dienst, Zentrale Geschätsstelle Bonn, Fakultät für Wirtschafts-und Sozial Wissenschaften, Lehrstuhl für Internationale Politik, Universidad de Postdam

PAISES BAJOS (6 entidades): Universidad de Amsterdam, International Institute os Social Studies, Erasmus University Rotterdam, Centre for Conflict Studies, Centro Internacional Agua Potable y Saneamiento, Ecologic-STRO

DINAMARCA (1 entidad): DIGNITY

PORTUGAL ( 3 entidades): The European Monitoring Centre for Drugs and Drug Addiction EMCDDA, Instituto de Ciencias Sociales, Universidad de Lisboa (Centro de Investigación).

FRANCIA ( 3 entidades): Instituto de Altos Estudios de Paris IHEAL-CREDAL, CIEPS.ISSN Intemational Centre, Instituto de Altos Estudios Sociales de Paris

SUIZA (1 entidad): Small Arms Survey

SUECIA ( 3 entidades): Universidad de Gottinghem, Stockolm International Peace Research Institute SIPRI, University of Gothenburg

NORUEGA (1 entidad): Instituto de Investigación, Fridtjof Nansen Institute

BÉLGICA (1 entidad): Carnegie Endowment for International Peace

ITALIA (1 entidad): Universidádegli Studi di Torino, Facoltá di Scienze Politiche

REINO UNIDO ( 6 entidades): FMR en Oxford, Forced Migration Review, Refugee Studies

Centre, Oxford Department of International Development School of Social and International Studies, University of Bradford, The International Institute for Strategic Studies, Loughborough University

CANADA (2 entidades): Real Policia Montada de Canadá, CIPC 
Propuesta de conversión del Centro de Investigación Científica (CINC) en Centro de Investigación Científica...

\section{Centro de Investigación Cientifica CINC 2015}

ESTADOS UNIDOS (32 entidades): National Defense University, Chigaco Police Departament, IGCC Publications and Web Site Content, Council of Foreign Relations New York Office, The Library of Congress, WOLA Washington Office on Latin America, WK Kellogg Foundation, Instituto del Conflicto Global de Cooperación Division of Arts and Humanities. The Paul H. Nitze School of Advanced International Studies (SAIS), Institute For International Peace Studies, Program Global Governance Woodrow Wilson International Center for Scholars, Florida International University, Centro de Estudios Hemisféricos de Defensa, Brookings Institution Press, MIT Press Journals, Johns Hopkins University, Camegie Endowment for International Peace, Global Financial Integrity, Global Integrity, IGCC Research Director, USMEX Associate Director, Vanderbitt University. Center for Hemispheric Defense Studies National Defense University, Center for Civil-Military Relations, Department of Politics and International Relations FIU University Park, Universidad de Miami, Instituto Crisis Group, Universidad de la Florida, Banco Mundial, Wilson Center, Open Society Foundation, University of Tampa.

MÉXICO (18 entidades): Instituto de Investigaciones Sociales de la UNAM, El Colegio de México, FLACSO México, Unidad Académica Playa del Carmen, Universidad de Quintana Roo, Colegio de la Frontera del Norte, Fundación Ford, WK Kellogg Foundation (México Office), Colectivo de Análisis de la Seguridad con Democracia, MUCD México, Colegio de la Frontera del Sur, Centro de Investigación y Docencia Económicas CIDE, Escuela Nacional de Antropologia e Historia, Universidad Quintana Roo, INACIPE-México, Instututo de Pensamiento Estratégico IPEA, Academia Superior de Seguridad Pública de la Policia Federal de México, Instituto de Acción Cludadana de México, FLACSO México, Universidad Autónoma de México, Universidad Autónoma de Sinaloa.

BELICE (1 entidad): Departamento de Policia de Belice

GUATEMALA (6 entidades): CIPREVI, APNC, Policia Nacional Civil de Guatemala, Konrad Adenauer Stiftung, Instituto de Investigaciones Juridicas, Universidad Rafael Landivar.

HONDURAS (2 entidades): Academia Nacional de Honduras, Instituto Tecnologico Policial.

NICARAGUA (3 entidades): Grupo Civico Ética y Transparencia, Instituto de Estudios Estratégicos y Politicas Públicas, Academia de Policia Nicaragua.

COSTA RICA (7 entidades): Corte Interamericana de Derechos Humanos, Escuela Relaciones Internacionales, Universidad de Costa Rica, Sistema de Bibliotecas, Documentaciōn e Información, Ciudad Universitaria Rodrigo Facio, Academia de Policia Costa Rica.

PANAMÁ (3 entidades): SENAFRONT, Policia Nacional de Panamá, Academia de Policia Panamá

COLOMBIA (13 entidades): Policia Nacional de Colombia, Instituto Nacional Penitenciario y Carcelario INPEC, Centro de Investigación en Politica Criminal, Universidad Externado de Colombia, Instituto de Estudios Interdisciplinarios, Universidad de Cali, FES Colombia, EAFIT Medellin, Fundación Ideas para la Paz, Corporación Excelencia en la Justicia, CLAE-Colombia, Revista Criminalidad Centro de Investigaciones Criminológicas (DIJIN) Policia Nacional de Colombia, Fundación Friedrich Ebert Colombia.

ECUADOR (4 entidades): Universidad Central Quito, Policia Nacional del Ecuador, FLACSO Ecuador.

VENEZUELA (3 entidades): Venozolana de Televisión, Paz Activa, El Nacional Caracas.

PERÚ (4 entidades): Programa de Seguridad Pública UNLIREC, CEDPE, FES-Perú, Universidad Católica.

BOLIVIA (2 entidades): Beta Gama - S.A., CLAE-Bolivia.

ARGENTINA (11 entidades): FLACSO Argentina, Strategic Support, Universidad Quilmes, Secretaria de Seguridad, Red de Seguridad y Defensa de América Latina, Centro de Estudios 
sobre Genocidio. Universidad Nacional de Tres de Febrero, Instituto de Estudios Comparados en Ciencias Penales INECIP, Instituto Latinoamericano sobre Segunidad y Democracia ILSED, Escuela de Policia Local Preventiva de Mar del Plata, Asesoria Juridica del Congreso de la República de Argentina.

CHILE (10 entidades): Fundación Paz Ciudadana, Instituto de Estudios Internacionales, Centro de Estudios Estratégicos, Instituto de Seguridad Püblica y Politica Internacional CESC, INAP Instituto de Asuntos Públicos. Universidad de Chile, FLACSO Chile, Universidad de Valparaiso, CED, Centro de Estudios en Seguridad Ciudadana.

BRASIL (7 entidades): Universidad Federal de Rio Grande del Sur FRGS, Policia Civil Do

Estado de Sao Paulo, Instituto Sou Da Paz, Universidad de Brasilia, Universidad de Sao Paulo, Grupo de Trabajo, VIVA Rio.

PARAGUAY (1 entidad): Instituto de Estudios Comparados en Ciencias Penales y Sociales IPEA, INECIP

URUGUAY (1 entidad): UNESCO Uruguay Sector Ciencias Sociales y Humanos

ISRAEL (1 entidad): Jerusalem Center for Public Affairs

EGIPTO (1 entidadad): Embajada de España

AUSTRALIA (4 entidades): School of Humanities and Social Sciences, The University of Melbourne, Vision of Humanity, Centre for Peace And Conflict Studies

3. Indización internacional:

Ver anexo de evaluación de parámetros de gestion cientifica y edtitorial hecho por LATINDEX a la revista "Policia y Seguridad Pública".

\section{Estudios publicados: Experiencias e impactos.}

Se entenderá en esta categoria como a todas aquellas investigaciones cientificas y estudios estratégicos que han sido tomados en cuenta, valorados y utilizados para cumplir objetivos de diversa indole como los siguientes:

1. Convertirse en material de consulta privilegiado de primer nivel en organismos especializados de inteligencia en el Estado y otras instituciones en el extranjero.

Resultado: Los distintos números han sido solicitados para levantado de colecciones de consulta en comunidades de seguridad e inteligencia tan variadas como el Organismo de Inteligencia del Estado (OIE); el Centro de Inteligencia Policial (CIP); el Centro Antipandillas Transnacional (CAT); Subdirección Antipandillas (PNC) y otros similares.

A nivel internacional,los ejemplares han circulado hasta en el Centro de Inteligencia y Seguridad Nacional CISEN (México)

2- Como incremento de los acervos bibliográficos especializados de áreas de investigación en seguridad de distintas instituciones internacionales de seguridad y defensa.

Resultado: Destacan y constan por los registros y correspondencias recibidas entre otros las siguientes instituciones:

- Sistema de Bibliotecas, Documentación en información / Universidad de Costa Rica

- The Tampa University (Florida) Estados Unidos de America.

- FLACSO Argentina.

- Ibero-Amerikanisches Institute Preu Bischer Kulturbesitz, (Alemania).

- Instituto de Estudios Globales de Seguridad de Hamburgo, (Alemania).

- Instituto Iberoamericano - Fundación Patrimonio Cultural Prusiano (Alemania). 
Propuesta de conversión del Centro de Investigación Científica (CINC) en Centro de Investigación Científica...

3- Utilizados para el desarrollo del planeamiento urbano en áreas deprimidas y empobrecidas.

Resultado: Atendido el estudio: "Propuesta analifica de cinco tesis interpretativas de la violencia estructural y el ejercicio de gobemabilidad en materia de gestión de segunidad pública y prevención de violencia en el Municipio de Santa Tecla". Año 1, vol. 1, 2011. Por parte del Ministerio de Obras Públicas (MOP) para ampliación de Calle de los Chorros; y por la Alcaldia Municipal de Santa Tecla, para cambiar los trazos originales del Paseo El Carmen, y redirigir el planeo urbano en el sector norponiente del municipio.

4- Utilizados como insumo estratégico para el desarrollo de doctrina policial en el ámbito de Naciones Unidas, para el desarrollo del área critica de operaciones de mantenimiento de paz en contextos post conflicto; asi como también otras entidades que por distintos objetivos solicitaron otros estudios para los efectos de planeo de su trabajo y proyección de sus inversiones.

Resultado:

- "El armamento de las pandillas salvadoreñas: Análisis de su letalidad, poder de fuego e implicancias de seguridad". Año 4, vol. 1, 2014. Solicitado por Sistema de la Integración Centroamericana (SICA); también solicitado por la División de Armas y Explosivos de la Policia Nacional Civil (PNC).

- "Las pandillas salvadoreñas y su comportamiento delictivo: Prospectiva de sus formas organizativas y expansión territorial para el proximo decenio 2015-2025'. Año 4, vol. 2, 2014. solicitado por el Instituto de Estudios Politicos e Internacionales de la Universidad de Colombia y por Cooperación Japonesa (JICA), para el planeo de sus inversiones en la zona oriental del pais para los próximos cinco años.

- "El policia estratégico frente a la crisis futura y las paradojas del Estado en la reconstrucción de su autoridad". Año 3, vol. 1, 2013. Solicitado por la Misión Diplomática Salvadoreña con representación en las Naciones Unidas. Además se ocupó como literatura obligatoria de lectura en seminarios de especialización de los mandos estratégicos de la Policia Nacional de Colombia, patrocinados por la National Defense University (Estados Unidos de América).

5- Utilizados como lecturas de estudio en carácter obligatorio para la formación del mando estratégico policial en otros paises [en la República de Colombia; en el marco de seminarios especializados de transferencia de capacidades patrocinados por la National Defense University]. También para adiestramiento de nivel básico en policia de nivel básico, como es el caso de Panamá.

6- Para retroalimentación de programas de investigación y comunidades de expertos en el àrea de seguridad y asuntos latinoamericanos.

7- Para ruptura de paradigmas de seguridad, situacion que ha generado la intersección cualitativa de los mundos académicos y los campos disciplinarios de la Estrategia y la Seguridad. 


\section{Cuadro (5)}

Tipo de investigaciones y estudios publicados en la revista "Policia y Seguridad Pública" (2011-2015)

\begin{tabular}{|c|c|c|c|c|c|c|c|c|c|}
\hline Tipolinvestigacion & $\begin{array}{c}\text { Rev. } \\
\text { (1) } \\
2011\end{array}$ & $\begin{array}{c}\text { Rev. } \\
(2) \\
2012\end{array}$ & $\begin{array}{c}\text { Rev. } \\
\text { (3) } \\
2013\end{array}$ & $\begin{array}{c}\text { Rev. } \\
\text { (4) } \\
2013\end{array}$ & $\begin{array}{c}\text { Rev. } \\
\text { (5) } \\
2014\end{array}$ & $\begin{array}{c}\text { Rev. } \\
(6) \\
2014\end{array}$ & $\begin{array}{c}\text { Rev. } \\
(7) \\
2014\end{array}$ & Total & Porcentaje \\
\hline $\begin{array}{l}\text { Articulb cientifico } \\
\text { lacademico? }\end{array}$ & 3 & 5 & 3 & 3 & 5 & 3 & 2 & 24 & $47 \%$ \\
\hline Eshudio estratogicol & 2 & 2 & - & 2 & 3 & $\cdot$ & 4 & 13 & $25 \%$ \\
\hline Informe estraiegics" & 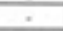 & 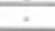 & 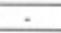 & - & . &. &. & $\cdot$ & $\cdot$ \\
\hline Balance estrategico 10 & - & . & - & + & . &. & . & . & $\cdot$ \\
\hline Estudio historiografico & 2 & - & 1 & 1 & * & - & 2 & 6 & $12 \%$ \\
\hline Estudio prospectivo" & . & . & . & 1 & - & 5 & 2 & 8 & $16 \%$ \\
\hline Total & 7 & 7 & 4 & $5^{\circ}$ & 8 & 8 & 10 & 51 & $100 \%$ \\
\hline
\end{tabular}

Fuente: Elaboracion propia en base a los registros institucionales del CINC.ANSP

"El total de imestigaciones de este número fue de (6). pero una de ellas presenta perfles de estudio estratigico y prospectivo. Por ello so especifica asi en la casilla.

Nota importante: Este cuadro fue incluido en un estudio publicado en la revista \# 6 sobre "Prospectiva de Seguridad", pero en su momento el muestreo de análisis abarcó hasta la revista \# 5. He aqui la estadistica actualizada hasta el recientemente publicado \# 7 sobre "Pandillas y Extorsiones". Lo destacable acá se refiere al aumento significativo de los estudios estratégicos, que incluye los de indole prospectivo y rondarian el $41 \%$ de la producción total. Una tendencia que constituye un indicador importante y de mucho futuro, si se considera la esencia de la presente propuesta.

8. Para inauguración, desarrollo pionero y difusión de la cultura de inteligencia, cultura prospectiva y cultura estratégica hacia los ámbitos del quehacer de las comunidades de seguridad y la investigación cientifica en el rubro.

El Centro de Investigación Cientifica ha establecido comunicación con diversas entidades académicas y tanques de pensamiento, tanto públicos como privados. Lo anterior como producto del envio sostenido de ejemplares fisicos de cada número y que ha contribuido a

\footnotetext{
'Son todos aquellos elaborados con la estructura IMRD (Introduccion, materiales/ método, resultados y discusida). Apoyadas en las distintas disciplinas clisicas del mundo chil tales como: has ciencias sociales, ciencias exactas y ciencias naturales o biomkdicas

${ }^{8}$ Referidos a imvestigaciones apoyadas en andlisis y perspectivas no convencionales, especificansente disciplinas cientificas prevalecientes en los ambitos de la seguridad y la defensa, que incluyen entre otras a la estrategia, gropolitica, imeligencia. prospectiva, etc.

"En la dedaratoria de arbitraje y clasificación de trabajos de la revista "Policia y Seguridad Pablica" del Centro de Investigkion Cientifica su definición reza ast: "Documentos originados en el ámbito de la burocracia publica, identificados como literatura gris; que es sibtematizada para un uso potencial y practico a través de su consamo por decisones y planificadores como su público meta primario y prioritario".

${ }^{10}$ De acuerdo al instrumento de la declaratoria de arbitraje ya mencionado, la definición teatualmeste dice: "Esfuerzos analiticos de tipo cuantitativo o cualitativo, que puede incluir como objeto legitimo de evaluación y estudio coyunturas, periodos determinados, fuerzas en presencia, fortalezas - debilidades (Estades, purtidos politicos, goleernantes. organizaciones y otros); hajo metodologias comparativas o de casos de estudio.

11 Estos son parte de los Estudios Estratégioss y conciernen a la exploración del futuro, a la evolexión prohable de las tendencias actuales; atienden para el caso particular de la produccion academica del Centro de Investigacion Cientificat la linea estratcyica neferida a "Estedies sobee el futuro y gestion de entomos coenplejos de seguridad" que esti declarada en la revista "Policia y Seguridad Publica".
} 
Propuesta de conversión del Centro de Investigación Científica (CINC) en Centro de Investigación Científica...

Centro de Investigación Cientifica CINC 2015

mantener un importante esfuerzo de distribución internacional. El listado de estas importantes instituciones, se detalla a continuación:

\section{Entidades internacionales}

- Instituto de Estudios Estratégicos de España.

- Center for Hemispheric Defense Studies 'William J. Perry" (Estados Unidos de América).

- Academia Nacional de Estudios Politicos y Estrategicos (Chile).

- Instituto de Estudios Politicos e Internacionales de la Universidad de Colombia (IEPRLI).

- Instituto de Investigaciones Sociales de la Universidad Nacional Autónoma de México.

- Escuela de Relaciones Intemacionales, Universidad de Costa Rica.

- Programa de Doctorado en Derecho / Universidad Francisco Landivar Guatemala.

- Instituto de Estudios Politicos y Estratégicos de Nicaragua.

- Centro Superior de Estudios de la Defensa Nacional (España).

- Instituto Iberoamericano - Fundación Patrimonio Cultural Prusiano (Alemania).

- Sistema de Bibliotecas, Documentación en información / Universidad de Costa Rica.

- FLACSO Néxico.

- Instituto de Estudios Internacionales Universidad Santiago de Chile.

- Instituto Vasco de Criminologia.

- National Defese University.

- European Monitoring Centre for Drugs and Drug Addiction Portugal.

- Instituto de Ciencias Sociales de Universidad de Lisboa, Portugal.

- FLACSO Argentina.

- Ibero-Amerikanisches Institute Preu Bischer Kulturbesitz (Alemania).

- Centro de Estudios y Cooperación Internacional (CECI).

- Ministerio de Defensa Nacional Colombia.

- The Tampa University, Florida (Estados Unidos de America).

- Instituto de Estudios Globales de Seguridad de Hamburgo, (Alemania).

\section{Retroalimentación de propuestas anteriores ${ }^{12}$.}

\section{Desafios estratégicos:}

1. "Politica de Investigación Cientifica institucional"

Se carece de este instrumento en la ANSP, no se ha planteado como linea estratégica en ningún plan quinquenal. Existen dos factores que han mediado e impactado negativamente en la posibilidad de desarrollo de este asunto. En primer lugar la falta de competencias profesionales y capacidades en la comunidad docente, algo que arrastra la escasez de perfiles estratégicos, además de problemas que conciemen al vacio de cultura de investigación y a la proliferación de modus vivendi negativos en la institución por parte de un significativo segmento del sector docente. $Y$ en segundo lugar, la omnipotente falsa

\footnotetext{
${ }^{12}$ Este apartado retoma todos los desafios estratégicos planteados en el informe intitulado: "Apuesto estrotelgica para el desarrollo futuro del Centro de investigación Cientifica CINC-ANSP". Lo hace desarrollando un planteamiento actualizado, situado a la altura de las nuevas circunstancias del entorno; pero todos, aspectos que en su origen continúan vigentes y constituyen imperativos de trabajo futuro. Documento estratégico presentado a la Dirección General el 30 de octubre de 2013. Consta de 16 páginas.
} 
creencia, que es con la constitución del Instituto de Educación Superior, la condición sine qua nom, por la cual puede generarse éstas politicas.

Esto equivale a esperar a contar con recursos para comprometer esfuerzos de construcción por algo y orientarlos luego hacia objetivos substantivos.

\section{2. "Sistema de Investigación Institucional"}

Mucho más complejo de montar si se carece de cultura de investigación y de un instrumento real y objetivo: La politica de investigación. En un sistema de investigación institucional, caben los diseños ad hoc de unidades especializadas para la producción cientifica; la conducción de comunidad académica, la construcción de presupuestos sostenidos y los niveles de investigación entre otras orientaciones.

Es muy probable que cualquier baremo para investigadores que se requiera instituir, presente brechas notables para su funcionalidad y supervivencia. El perfil colectivo de la comunidad docente actualmente dista de los imperativos y estándares requeridos para la modernización educativa en el ámbito policial.

\section{3. "Agenda y Plan de Investigación y Analisis"}

A excepción del CINC que lo ha adoptado como cultura práctica. No existen ni programas de investigación, ni agenda en la ANSP. A pesar que se ha insistido en varios espacios de discusión, de la necesidad de adoptarlo en la comunidad docente y en quienes deben liderar ese particular asunto y que conciernen a los esfuerzos de planeamiento educativo. No se ha considerado una buena experiencia en esta materia y se han menospreciado las buenas prácticas derivadas de la misma.

\section{4. "Programas de Investigación en Seguridad"}

Ocurre una situación similar a 10 anteriormente expuesto. Solamente se hace hincapié de que no se trata de un programa funcionando bajo requisitos estrictamente de carácter administrativo; sino de un reservorio de contenidos estratégicos que desarrollan lineas de trabajo, contribuyen a la reducción de brechas en materia de investigación y aportan al desarrollo de aristas y dimensiones de análisis ausentes y escasas en muchos ambientes institucionales y propios del ámbito de seguridad.

\section{5. "Relacionamiento Estratégico"}

Importante para un ente que prosiga por la senda de la especialización en los "Estudios de Seguridad"; profundizar el concepto de cooperación academica y cientifica bajo las modalidades de alianza internacional y la del asocio estratégico público -público. En el primer caso plantea desafios de transitar a una cultura editorial bilingūe 0 multilingüe, estrechar vinculos con centros de investigación y participar en coinversiones e iniciativas de investigación. En el caso del segundo plantea que las distintas iniciativas y entes del sector público dedicadas a la investigación cientifica, aporten a los "Estudios de Seguridad" $\infty$ n otras perspectivas disciplinarias y se generen espacios para la coparticipación en proyectos avanzados de carácter transversal para el Estado.

\section{6. "Indizaciones especializadas"}

Se ha logrado seis indizaciones, pero existe el desafio de alcanzar igual cantidad para los años venideros. Esta estrategia de incorporación de los haberes de investigación en bases de datos especializadas, pretende extenderse a otros sitios como Hapi, Ebsco, Scielo, 


\section{Centro de Investigación Científica CINC 2015}

Redalyc y plantearse el reto de avanzar hacia la corriente principal de la ciencia (Science Index Citation). Cada indización se logra, comprometiendo una candidatura fuerte, cumpliendo parámetros, atendiendo compromisos nacionales e internacionales y dando estabilidad a las politicas editoriales y reglas del juego en el proyecto.

\section{7. "Planta de investigadores y comunidad de especialistas"}

Sin inversión en investigación para la seguridad, es complicado que el proyecto sea sostenible. No se puede pretender más productos con menos; y aunque la experiencia reciente parezca demostrarlo; continuando de esa manera, constituye un camino seguro al estancamiento y al hecho de que indizaciones más especializadas dejen de prosperar y la innovación editorial deje de avanzar por sendas de desarrollo constante. Sin planta de investigadores para aseguranza de la participación, en el tercio requerido para producción cientifica e incluso su remuneración adecuada, se puede lograr cualquier cosa, menos una situación estable basada en la alta identificación de los miembros hacia metas y retos mayores y por ende en su movilización hacia el rol de construcción pública de ideas de avanzada y estuerzos rompe paradigmas.

\section{8. "Umbral económico y salarial"}

Tanto en la perspectiva de conversión de un CINC en un CINCEES, cómo de una modalidad de fortalecimiento cualitativo de un CINC. Es imperativo el establecimiento de nuevos rangos de plazas. Las existentes cuentan con techos económicos no competitivos y que vulnera el concepto de estimulo o incentivo para desempeños laborales más prolongados en el tiempo.

La estrategia acá debe partir de la base de dignificación económica de un equipo de trabajo y la propuesta de una nueva frontera salarial que traiga consigo mayores umbrales de realización personal y profesional para los miembros.

\section{9. "Nuevos productos de investigación"}

Parte de las credenciales académicas de un tanque de pensamiento o centro de investigación descansan en el grado de asertividad, influencia en áreas criticas y uso potencial de su producción cientifica. Por ello la diversificación de productos avanzados, su circulación entre los públicos meta estratégicos que se han concebido y la efectividad en la distribución internacional de los mismos; son aspectos indisolubles y vinculados entre si. El contacto con nuevos evaluadores, constituye la plataforma de expansión de las redes profesionales y de expertos para la cooperación académica; estimulario resulta de orden fundamental. Hacerlo responsablemente también es importante y ello implica, el igual concurso de argumentos solidos y los distintos paradigmas en los que se apoya la investigación apegados a los formatos cientificos de las distintas comunidades de especialistas.

\section{0. "Gestión de información y documentos"}

Un gestor de la investigación en seguridad, debe desarrollar el componente de la información virtual y los centros de documentación. El CINC cuenta con un Centro de Documentación e Información (CEDIN); pero el techo para que este cuente con colecciones especializadas en su haber de literatura, pasa por considerar y tratar tres aspectos de relevancia. Por una parte identificar las necesidades de la Academia Nacional de Seguridad Pública (ANSP) y la Policia Nacional Civil (PNC) en matera de requerimientos educativos, de 

(CINC) en Centro de Investigación Científica...

Centro de Investigación Cientifica CINC 2015

materias especializadas y de sistemas de trabajo. En segundo lugar de habilitación de mecanismos de compra virtual, conductos del comercio electrónico, que posibiliten el acceso a la literatura muy especializada y actualizada, que de momento no puede ser cubierta por ninguna oferta nacional. $Y$ en tercer lugar inversiones estratégicas significativas en la plataforma informática que a la fecha es totalmente ineficaz. No sólo cuenta con una conexión lenta, sino que es inoperante para los menesteres de enlace con otras bibliotecas virtuales. La inestabilidad de la red interna conlleva que en diversas ocasiones, las publicaciones de "Policia y Seguridad Pública" no se hayan alimentado para las distintas bases de datos; por problemas de origen en los servidores de la ANSP. Situación inaceptable, debido a la ausencia de una politica de desarrollo informático institucional.

Cuadro (6)

Consolidado del Centro de Documentación e Información (CEDIN) (hasta Julio 2015)

\begin{tabular}{|c|c|c|c|c|c|}
\hline \multirow{2}{*}{ 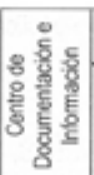 } & Préstamos & $\begin{array}{c}\text { Bibliografia } \\
\text { impresa }\end{array}$ & $\begin{array}{c}\text { Bbliografia } \\
\text { Digital }\end{array}$ & Audiovisuales & Tesis ${ }^{*}$ \\
\hline & 786 & 946 Títulos & $\begin{array}{c}5,000 \\
\text { archivos } \\
\text { periodisticos }\end{array}$ & 83 & 98 \\
\hline
\end{tabular}

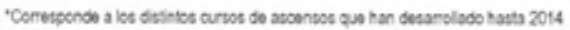




\title{
VII. CONTRAPROPUESTA RELACIONADA AL ÁREA DE INVESTIGACIÓN CIENTIFICA EN EL MARCO DE LA REESTRUCTURACIÓN ORGANIZATIVA DE LA ANSP Y SU CONTEXTO DE CONVERSION A INSTITUTO DE EDUCACIÓN SUPERIOR (IES) ${ }^{13}$
}

\begin{abstract}
Propuesta fuerte:
Propuesta 1: El Centro de Investigación Cientifica debe contar con un estatuto de autonomia que le permita mayor margen de desarrollo para alimentar sus lineas de trabajo basadas en la proyección extra institucional e internacional. Una especie de equivalente a lo que el IUDOP (Instituto de Opinión Pública) tene en la Universidad Centroamericana; el ICTI (Instituto de Ciencia, Tecnologia e Innovación) en la UFG; y el Centro de Politicas Públicas de la ESEN (Escuela Superior de Economia y Negocios). Estos últimos son una especie de tanques de pensamiento y tienen sus programas de investigación y su agenda; pero no solamente atienden las prioridades institucionales de la universidad, sino tambièn un ènfasis de trabajo fundamental hacia los problemas, áreas criticas y materias especificas que les dieron origen como entidades. En cuanto al sector público existen otras fórmulas y figuras de entidades de educación superior como el CAEE (Colegio de Altos Estudios Estratégicos) y el IEESFORD (Instituto Especializado de Educación Superior para la Formación Diplomática); ambas están vinculadas a estructuras ministeriales de los sectores de Defensa y Relaciones Exteriores, su labor fuerte se basa en una oferta educativa de nivel de postgrado pero la proyección social y la investigación politico estratégica propia de este tipo de entes ha tenido poco desarrollo y constituye su déficit principal. Porque también arrastran la falla de origen, que concierne a ser evaluadas como universidades, cuando en estricto sentido no lo son.
\end{abstract}

En el caso del CINC por haber desarrollado un tipo de investigación de nivel politico estratégico, debe ser mejor financiado y reforzado en planta, establecido y ubicado en un ámbito dependiente de la máxima unidad jerárquica de la estructura (Dirección General y Sub Dirección Ejecutiva). Por supuesto que este se apoya en agenda y programas que es lo que no existe en el sector docente y donde se quiere propiciar otro tipo de investigación más básica. En el marco de esta propuesta que nos parece la más vanguardista y prudente, el CINC debe convertirse en una unidad de staff, especificamente un Centro de Investigación Cientifica y de Estudios Estratégicos de Seguridad (CINCEES), recogiendo la agenda de desarrollo que ya se presentó en una propuesta pasada. No hacerlo implicaria que los riesgos de la inercia institucional, del burocratismo y la falta de visión estratégica propios de los cambios de timón, los relevos de los liderazgos ó las reformas mal concebidas y conducidas, se incrementen y terminen ahogando esta iniciativa que ha sido sumamente avanzada en su género. Esta propuesta constituye el seguro de vida de esta experiencia, la garantia de su permanencia como blindaje

\footnotetext{
13 La presente sección consthuye el texto integro y mejorado de un documento de dimensiones regulares, que el Centro de Investigación Clentifica, a través de su coordinador principat; entregó a personal de la Unidad de Planificaoión Instlucional (UPI), como insumo base preliminar. El propósito del mismo se refirio a enriquecer la concepción general y el stock de propuestas internas en torno a la funcion estratbgica de la inwestigación cientifica. Remitido el mierconles 15 de abril de 2015, por via de correo electrónico.
} 
preventivo contra toda iniciativa que socave su desarrollo. Además es imperativo que funcione y se expanda con el formato que se propone, porque el entorno de sequridad es complejo y en ninquna entidad del Estado se generan estos productos diseñados $y$ concebidos para los decisores del ámbito público, en este caso del campo de la seguridad. Todo 10 anteriormente planteado en esta propuesta constituye la sustancia del concepto estratégico en el que debe modelarse el futuro del CINC. Recuérdese que a nivel de politicas editoriales, objetivos estratégicos y concepto estratégico de investigación; el vinculo orgánico es hacia la comunidad de seguridad y entre los impactos esperados es la incidencia fundamental en el fortalecimiento del aparato policial y de seguridad, las politicas públicas del sector y la educación policial en el nivel superior de postgrados. Los aspectos antes mencionados surgen del imperativo de fomento de la cultura de investigación para superar los graves problemas del pais en materia de seguridad pública y por lo tanto el proyecto institucional CINC ha contribuido a llenar el vacio existente en materia de estudios estratégicos de seguridad, esfuerzo que debe continuar.

Propiciar condiciones para el desarrollo del CINC y su conversión progresiva en un tanque de pensamiento para la seguridad, consideramos es la opción más inteligente $y$ avanzada con la cual la ANSP puede capitalizar la experiencia y los logros del CINC y por lo tanto propiciaria un umbral para su potenciación.

\section{Crítica a la propuesta de la División de Estudios.}

En el caso de que se plantee una División de Investigación y Proyeccion Social o su variante una Sub Dirección de Investigación y Desarrollo, este modelo presenta los siguientes inconvenientes:

A. Un departamento de difusión y divulgación únicamente contribuiria a la burocratización de esta entidad pública. Siendo esta función básica una responsabilidad de la estructura institucional en su conjunto en su labor de apoyos logisticos y otros menesteres inherentes a la divulgación. Lo más probable es que los ciclos de trabajo en estas funciones especificas se reducirian a dos por año, trayendo como consecuencia la subutilización de personal y su respectiva carga salarial. En definitiva no es consecuente con un verdadero mecanismo institucional que solvente aspectos de logistica y coordinaciones. A manera de ejemplo, de acuerdo a la experiencia del CINC los públicos meta de sus producciones cientificas se encuentran en las altas esferas del Estado, especificamente las comunidades de seguridad, defensa, inteligencia y relaciones exteriores; esto plantea un esfuerzo extra institucional de divulgación y distribución, requiere el concurso del más alto nivel para efectos de establecimiento de contactos, enlaces y puentes para llegar a los respectivos destinatarios. No es una cuestion que conciema únicamente al CINC sino que concieme a la organización institucional pues es una expresión de su rostro, imagen y diplomacia hacia afuera.

B. El formato adoptado para la conversión de la ANSP en un Instituto de Educación Superior (IES), recoge por la influencia de la Ley General de Educación Superior las funciones tradicionales de toda universidad referidas a docencia, investigación cientifica y proyección 


\section{Centro de Investigación Científica CINC 2015}

social. A pesar de que la ANSP no es una universidad y arrastra los defectos de fábrica originados en la ley y agravados por las concepciones imperantes tanto en los pares evaluadores del Ministerio de Educación (MNED) y la comunidad al interior de la ANSP. Nos parece desacertado reducir la proyección social a una unidad dependiente de un supuesto ente rector de la investigación en la institución. De tal forma que recomendamos que no sea incluida como área subordinada y que tampoco emplee la fórmula de fusión de ambas funciones estratégicas; sobre todo si se considera que es sumamente exigua en recursos y endeble en planta de personal especializado. En muchos casos reales la fusión de ambas funciones se ha mostrado inoperante y desastrosa y por lo general ha planteado el desarrollo desigual de las mismas, puesto que la proyección social es algo que involucra a la organización, que crea sus propios requerimientos, establece sus vasos vinculantes y de comunicación con el entomo y los actores que en él se desenvuelven y que se asumen como públicos prioritarios.

C. No es prudente ni razonable incluir dos o más tipos de investigación en una misma estructura. Por ejemplo propiciar la cohabitación entre esfuerzos de investigación politico estratégica con otras más de tipo educativo-curricular. Lo anterior se debe a muchos factores criticos tales como: La existencia de dos sedes fisicas y su consiguiente impacto en la fragmentación organizacional; las funciones y roles que desarrollan docentes e investigadores en sus propios ámbitos y comunidades son distintas en esencia, puesto que unos carecen de práctica docente y otros de experiencia sistemática en materia de investigación y el levantado de estudios cientificos. De tal manera que lo más factible es que un tipo de investigación de nivel educativo-institucional debe asimilarse en la estructura de la División de Estudios que constituye el nicho base de la oferta y praxis educativa, y a la que es lógico que esté vinculada. Por ello es contraproducente que la misma exista como un componente parte de una División de Investigación y Proyección Social; corriendo el riesgo en el proceso de mostrarse aislados del proceso educativo. Lo anterior estaria planteando la desmovilización de una mega estructura, que en caso de existir tendria que generar cinco subproductos de gran importancia, entre estos: 1) Sistema de investigación institucional; 2) Polifica de investigación institucional; 3) Agenda de investigación institucional; 4) Programas de investigación; 5) Lineas estratégicas de investigación. Estos componentes serian de rigor, porque conllevan a la activación de esfuerzos principales, niveles de investigación, actores responsables, publicaciones especializadas, públicos meta estratégicos y condiciones para el planeamiento, la verificación y el control.

\section{Conclusión:}

No es procedente ninguna modalidad de División de Investigación y Proyección Social en el diseño examinado, más aún si para ello pretende absorber la experiencia exitosa del Centro de Investigación Cientifica CINC-ANSP. Asi como también constituiria una via ruinosa la asimilación del CINC dentro de una estructura mayor, en este caso la División de Estudios. 
En el caso de la primera modalidad apuntada, pudiese desarrollarse si y solo si se cumplen dos condiciones fundamentales:

1. Su no dependencia jerárquica de la Division de Estudios, y si por el contrario de la Sub Dirección Ejecutiva y la Dirección General.

2. La decisión politica, legal $e$ institucional que habilite la conversión del Centro de Investigación Cientifica CINC en Centro de Investigación Cientifica y Estudios Estratégicos de Seguridad (CINCEES). Esta estructura puede existir en simultáneo y en el marco de un ámbito claro de diferenciación con una División de Investigación y Proyección Social. La insistencia en una investigación de nivel básico curricular-educativa, debe ser concebida, desarrollada y liderada por la comunidad docente, que es la que se inscribe en la cotidianidad de esos menesteres y propiciar asi su propia experiencia. 